\title{
Indulgent foods can paradoxically promote disciplined dietary choices
}

\author{
Nicolette J. Sullivan, Gavan J. Fitzsimons, Michael Platt, Scott A. Huettel
}

Accepted, Psychological Science

\begin{abstract}
As obesity rates continue to rise, interventions promoting healthy choices will become increasingly important. Participants ( $\mathrm{N}=79)$ made binary choices between familiar foods; some trials contained a common consequence (CC) that had a constant probability of receipt regardless of the participant's choice. We theorized - based on simulations using a value normalization model - that indulgent CCs potentiated disciplined choices by shaping other options' perceived healthfulness and tastiness. Our experimental results confirmed these predictions: an indulgent $\mathrm{CC}$ more than doubled the rate of disciplined choices. We used eye gaze data to provide insights into the underlying mechanisms, finding that an indulgent $\mathrm{CC}$ biased eye gaze toward healthy foods. Furthermore, attention toward the common consequence predicted individual differences in behavioral bias. Results replicated across two independent samples receiving distinct goal primes. These results demonstrate that introducing an irrelevant indulgent food can alter processing of healthier items - and thus promote disciplined choices.
\end{abstract}




\section{Introduction}

Global obesity rates have doubled since 1980 , with $40 \%$ of world adults estimated to be overweight or obese (World Health Organization, 2016). Because caloric intake is the major risk factor for obesity (Rosenheck, 2008), researchers and policy makers have attempted interventions to increase dietary self-control and encourage disciplined food choices. Yet, such interventions have had only limited success. For example, the introduction of healthier options to a major fast-food chain's menus did not lead to a concomitant increase in the selection of healthful options (Deng, 2009; Jargon, 2013); more generally, the disclosure of calorie information on menus has had only a modest influence on mean calorie intake (Dumanovsky et al., 2011). Furthermore, local initiatives to increase the number of healthful options in "food deserts" have had little to no effect on dietary intake, foods in the home, or consumers' body mass index (Cummins, Flint, \& Matthews, 2014; Elbel et al., 2015; Morland, Wing, \& Diez Roux, 2002; Seymour, Yaroch, Serdula, Blanck, \& Khan, 2004).

Why might increasing the availability of healthful foods have such limited effects on dietary choice? One intriguing possible explanation is that introducing new foods to a choice set regardless of whether they are desirable - changes how consumers perceive the healthfulness and taste of other available foods. Decision biases associated with the set of options available for choice are often referred to under the umbrella term "context effects" (e.g., Chakravarti, 1983; Payne, 1982; Prelec, Wernerfelt, \& Zettelmeyer, 1997; Rooderkerk, Van Heerde, \& Bijmolt, 2011). Context effects have been demonstrated in tasks ranging from simple perceptual decisions to complex financial choices and can lead laboratory participants and consumers to make choices inconsistent with their preferences (Huber, Payne, \& Puto, 1982; Trueblood, Brown, Heathcote, $\&$ Busemeyer, 2013). For example, the addition of a healthful option to a choice set can counterintuitively result in more indulgent preferences among the other options, particularly for individuals with high trait self-control (Wilcox, Vallen, Block, \& Fitzsimons, 2009). These results suggest that simply increasing the availability of healthful foods - an approach adopted by many interventions - could have unintended negative consequences for dietary choice.

Here, we examine how the introduction of a new food option alters self-control in dietary choices, using an approach that precludes that option from having any objective impact on participants' outcomes. Participants made a series of incentive-compatible choices between familiar foods. In the Baseline task, only two foods were present on the screen - and the participant's choice determined which item might be received for later consumption. In the key Common Consequence (CC) task, however, we paired each of those two foods with the same third food within a gamble (Figure 1), so that participants had a 50\% chance of receiving the common consequence (regardless of what they chose) and a 50\% chance of receiving their chosen food. Importantly, a rational decision maker should ignore any probabilistic consequence common to all choices, such that it should not influence preferences over other non-common potential outcomes (Kahneman \& Tversky, 1979; Savage, 1954). Yet, common consequences do often influence choice (e.g., Allais, 1953; Kahneman \& Tversky, 1979; Starmer, 1992), although the mechanisms behind those behavioral effects remain largely unknown.

We use the introduction of a common consequence to perturb the processes of dietary choice while controlling for objective features of the decision - and while concurrently measuring both choice behavior and moment-to-moment information acquisition using eye-tracking. We constructed participant-specific choice sets using their idiosyncratic ratings on the canonical dimensions of taste and health (Figure 1a). Some items were rated as healthy but not tasty; we 
label these as "disciplined" options. Other items were tasty but not healthy; these reflect "indulgent" options. We compared trials involving the same objective choice in three different contexts: a simple binary choice between the two foods (Baseline; Figure 1b), in 50-50 food gambles paired with a disciplined common consequence (Common Disciplined, CD; Figure 1c), or paired with an indulgent common consequence (Common Indulgent, CI; Figure 1d). Importantly, the common consequence should be ignored such that participants' choices should be identical across all three contexts (i.e., in Figure 1b-d, reflecting only their preferences between salmon and Oreos). Yet, as we show throughout this paper, the nature of the common consequence exerts dramatic effects on choice behavior within and across individuals, on the parameters of decision models, and on measures of attention obtained through eye-tracking.

A

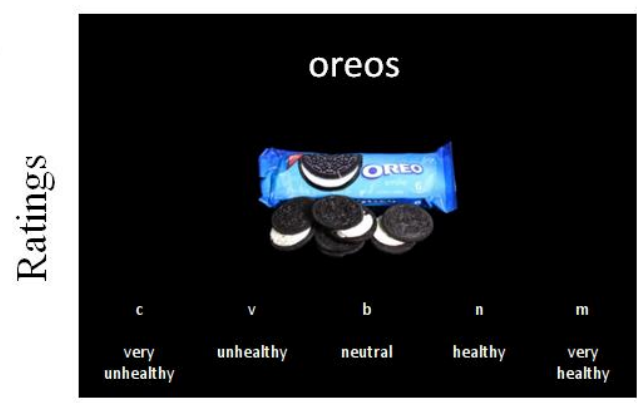

C

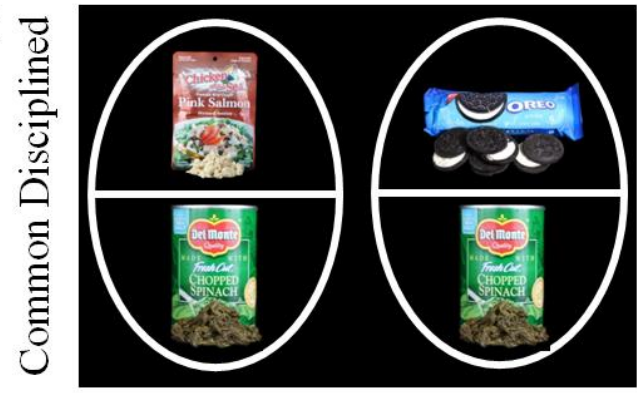

B
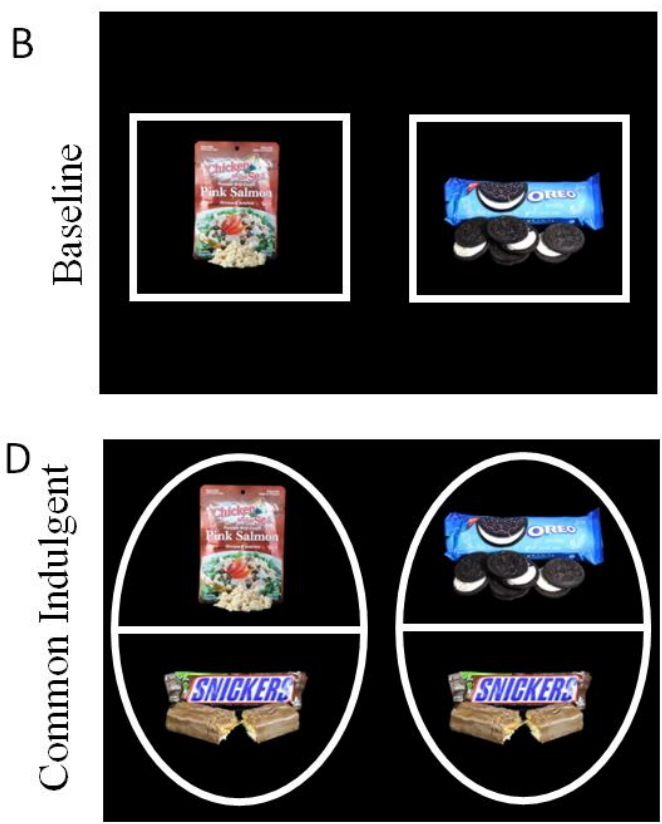

Fig. 1. Experimental tasks. In the food ratings task (a), participants indicated the perceived taste, perceived healthfulness, and desire for consumption (i.e., "wanting") of foods on five-point scales; shown here is a rating screen for healthfulness. In the Baseline task (b), participants made choices between two foods. In the Common Consequence (CC) task, each trial consisted of a binary choice between gambles containing a pair of foods. If the participant selected the left gamble, they would have an equal chance of receiving either one of the two foods in the circle. Example (c) shows a healthy but not tasty common consequence ("Common Disciplined" trials; CD). Example (d) features a tasty but not healthy common consequence ("Common Indulgent" trials; CI). Because the participant has the same probability of receiving the common consequence regardless of their choice, the selection of the left or right gamble should only depend on the non-matched items. 


\section{Methods}

Young adult participants completed four experimental procedures in a fixed order: a food ratings task, health/taste goal priming, incentive-compatible choices in the Baseline task, and incentivecompatible choices in the Common Consequence task. All experimental stimuli were presented using the Psychophysics Toolbox for MATLAB (Brainard, 1997) and are available online along with the experimental data. At the end of the experiment, participants received one of their chosen food items for immediate consumption.

Participants. Seventy-nine young adults from the Durham-Chapel Hill community (63\% female; mean age 24.4 years $(\mathrm{SD}=8.1$ years $)$ participated in this 90 -minute study. The targeted sample size (40 individuals in each of two priming groups) was determined based on measurements in two independent datasets (results in preparation for publication) that included a binary choice task similar to our Baseline task described below. First, we calculated the effect of our differential priming conditions on the proportion of healthy choices across a large sample of subjects $(\mathrm{N}=133)$, which generated an approximate required sample size of between 40 and 45 participants in each prime group (via the sampsizepwr function in MATLAB). We next examined the robustness of our priming effects in a second independent data set $(\mathrm{N}=40)$, in which the main effect of our primes fully replicated. Based on these prior results, we set 40 participants in each prime group as the target sample size in the current study. We note that the current study includes the novel Common Consequence condition that has not been previously reported in the literature; accordingly, we used the theorized effects of priming to estimate the sample size needed for each of our prime groups, and then used the two priming conditions as independent replications of the Common Consequence effects.

Participants were screened for any dietary restrictions. Prior to the experiment, participants fasted for four hours, with compliance documented by computerized self-report. Of these 79 subjects, four subjects were excluded because of insufficient eye gaze data and two subjects were excluded due to insufficient variance in their food ratings to create an adequate number of trials for each condition. Participants were compensated with $\$ 12$ in cash and a snack food eaten at the end of the experiment. All participants gave informed consent under a protocol approved by the Duke University Institutional Review Board.

Food Rating task. Participants first rated all of the experimental foods on three five-point scales: tastiness, healthfulness, and wanting (see Supplemental Methods). Scale type, food presentation order, and scale left-to-right direction were randomized across participants. Stimuli were $600 \mathrm{x}$ 600 pixel full-color images, presented alongside a one- to three-word item name (e.g. "Oreos"). All photographs of foods were taken under controlled lighting conditions, used similar positioning and sizing, and included a sample of the food outside of its packaging (e.g., showed chips outside of the chips bag).

Goal Priming. Then, as part of a larger, multiple-experiment examination of dietary nudges, all participants were randomly assigned to one of two priming groups. After the ratings task, participants read a short script (see Supplemental Methods) that described the importance of eating either healthier foods ("Health Prime"; N=40), or tasty foods ("Taste Prime"; N=39). We treat these two groups as independent replication samples in the current experiment; that is, we show that our effects are present in both samples and are robust to differences in participants' health goals. 
Baseline task. Afterward, participants performed 300 binary choices between pairs of the foods they had rated in the previous task. Half of the trials were formulated such that one food was tastier and less healthy than the other food (Conflict trials). An array of all possible combinations of food pairs in which one food was tastier, and less healthy, than the other was created. Among those, 150 were selected at random. In cases where there were fewer than 150 possible combinations, some pairs were repeated, and the pairs that repeated were also chosen at random. In cases where there a participant only rated foods on one end of a scale (e.g., rated all foods as tasty, or all foods as unhealthy), food pairs were selected at random. This occurred for only one participant, who rated all task foods as "Very Tasty," the highest taste rating. This participant was not considered to have any Baseline Conflict trials, and was therefore not included in analyses using Baseline Conflict trial behaviors. One third of trials presented options using images, one third as their item names from the ratings task, and one third featured one option in words and the other as an image.

Presentation order was randomized across trials and participants, with the constraint that an item could not repeat within a five-trial window. In all results presented in this manuscript, we analyze data from the image-image conflict trials to match the properties of the Common Consequence task (described below); however, all reported results remain statistically significant and hold their direction of effect when combining data across all three visual presentation conditions and when combining across both conflict and non-conflict trials (see Supplemental Results).

Common Consequence task. In the fourth and final task, participants made 300 self-paced choices between two different food gambles, each containing two foods with equal chances of delivery. On the Common Consequence trials ( $\mathrm{CC} ; 2 / 3^{\text {rds }}$ of all trials), the two gambles each contained one food shared between the gambles (the common consequence), and one unique food each (see Figure 1c and d for examples).

We varied whether the matched $\mathrm{CC}$ food was rated by the participant as healthier than tasty (Common Disciplined trials; $\mathrm{CD} ; 75 \%$ of $\mathrm{CC}$ trials) or tastier than healthy (Common Indulgent trials; CI; $25 \%$ of CC trials). The imbalance of the number of CI and CD trials was motivated by Wilcox et al. (2009), who found that the introduction of a more disciplined food decreased the proportion of healthy choices. Given that result, we decided to create additional trials of the CD condition. The common consequence foods were selected by creating an array of all foods rated by the participant as tasty, but not healthy (CI trials), or healthy, but not tasty (CD trials). For each trial, one of these foods was selected randomly to be the common consequence, while ensuring that it was not either of the unique foods in the trial. The unique foods were selected using the same algorithm as used for Conflict trials in the Baseline task (see above).

On the remaining No Common Consequence trials (NCC; $1 / 3^{\text {rd }}$ of all trials), foods were selected for each trial in a similar manner, with one major difference. Instead of using an identical food for the common consequence to be shared between the two gambles, two foods were instead matched as closely as possible in their taste and health ratings. In cases where there were not two tasty, less healthy or healthy, less tasty foods that were equally matched in their ratings on the five-point scale, we matched them within one rating point. If there were still no foods, we matched them within two points. If there were still no foods available, we selected foods at random; this occurred for one participant. Half of the NCC trials were targeted to match the CD condition, and half were targeted to match the CI condition. This means that the CC and NCC 
trials were matched in the overall statistics of their ratings, such that any differences in behavior can be attributed to the presence of the common consequence.

Presentation order was randomized across trials and participants. Participants indicated their choice on each trial by pressing a button on a keyboard. Eight participants did not have sufficient variance in food ratings to allow for any $\mathrm{CD}$ or $\mathrm{CI}$ trials (e.g., they tended to rate all foods as very tasty and/or very healthy), and were excluded from analyses including $\mathrm{CI}$ and $\mathrm{CD}$ trials.

Incentive Compatibility. At the end of the experiment, one trial from either the Baseline or Common Consequence task was randomly selected for consumption. If the trial selected was from the Baseline task, participants received the food they selected on that trial. If the trial selected was from the Common Consequence task, participants were served one of the two foods in their selected option, randomly determined. Participants could leave immediately after eating one serving of the food, or could wait thirty minutes in the experiment room (78 of the 79 participants consumed the food).

Eye gaze collection and processing. Eye position data were collected during all tasks with a Tobii T60 (Stockholm, Sweden) remote eye tracking system at a temporal resolution of $60 \mathrm{~Hz}$ $(+/-.002 \mathrm{~Hz})$. Regions of interest (ROIs) were drawn around each food image and inflated by 50 pixels on each side to account for variability in gaze calibration. Total gaze time to each ROI was calculated for each trial and participant. Next, total dwell time on the common consequence and on each of the unique foods was calculated. We decided a priori to omit the final fixation from these calculations because it often co-occurs with the execution of a choice.

For each subject, a gaze bias score was calculated using their fixations in the Common Consequence task. First, gaze time to each food was converted to a percentage of total food item gaze time, to control for any differences in response times between tasks. Next, the gaze bias score was calculated by taking the difference in percentage gaze time to the unique healthy relative to unique tasty food.

Data analysis. All reported psychometric regression results use full mixed-effects regression methods. Specifically, we aggregated all individual choice data and regressed our predictor on the outcome variable as a fixed effect and added a subject indicator as a random effect. All mixed-effects regressions included random slopes and intercepts. All statistical analyses were performed in MATLAB. All statistical tests that resulted in a value less than $\mathrm{p}=1 \times 10^{-10}$ are reported at that level, given the limits on the precision of our statistical analyses. 


\section{Results}

Our analysis plan began with examination of the psychometric properties of participants' decisions, in order to confirm that participants' choices were consistent with their ratings of the foods. We next compared choices in the Baseline and Common Consequence tasks, which were matched for all objective features of the decision save for the introduction of the common consequences. Finding clear effects of the common consequences upon choices, we then used model simulations to explore whether those effects could be explained by a value normalization process - which in turn would generate predictions about changes in the decision weights associated with different attributes and about the process of information acquisition during choice. Finally, we examined whether individual differences in that information acquisition process could predict which participants were most biased by the common consequence.

\section{Psychometric properties of choice behavior}

We examined how choices and response time (RT) depended on trial-to-trial variation in relative value of the foods. Hereafter, we use "value" to refer to each participant's self-reported wanting (i.e., their desire to eat each food at the end of the experiment). Figure 2a shows the probability of choosing the left option on a trial as a function of the relative value advantage of the left option (measured by wanting left $_{\text {- }}$ wanting $_{\text {right }}$; this formula reflects the difference in the unique options on CC trials). To account for any possible differences in the range of food ratings between the two tasks, we normalized value differences for each task from -1 to 1 . We found the expected logistic relationship between choices and relative value for both the Baseline and Common Consequence tasks (Figure 2a; $\mathrm{CC}$ logistic slope $=4.22, \mathrm{t}(15776)=15.71,95 \% \mathrm{CI}=$ [3.69 4.75], $\mathrm{p}<1 \times 10^{-10}$; Baseline logistic slope $=4.83, \mathrm{t}(7098)=15.19,95 \% \mathrm{CI}=[4.215 .45], \mathrm{p}$ $\left.<1 \times 10^{-10}\right)$. See Supplemental Results for analyses of the influence of task and trial order on these results.

Response time was greatest for trials on which the two options were matched for subjective value, and decreased within increasing difference in subjective value; this quadratic function was observed for both the Baseline task (Figure 2b; quadratic slope $=-724.85, \mathrm{t}(7095)=80.33,95 \%$ $\mathrm{CI}=[-883.37-566.34], \mathrm{p}<1 \times 10^{-10}$ ) and for the Common Consequence task (quadratic slope = $\left.598.68, \mathrm{t}(15773)=108.70,95 \% \mathrm{CI}=[-486.13-486.13], \mathrm{p}<1 \times 10^{-10}\right)$. Response time was longer in the Common Consequence task, which may partially reflect the processing of additional items on the screen $\left(\mathrm{CC}\right.$ mean $=1828 \mathrm{~ms}$, Baseline mean $=1502 \mathrm{~ms} ; \mathrm{d}=0.52, \mathrm{t}(70)=7.23, \mathrm{p}=5 \times 10^{-}$ ${ }^{10}$ ), and there was a larger quadratic slope associated with relative subjective value in the CC than Baseline task $\left(d=0.49, t(70)=4.80,95 \% \mathrm{CI}=[73.80178 .56], \mathrm{p}=9 \times 10^{-6}\right)$. 

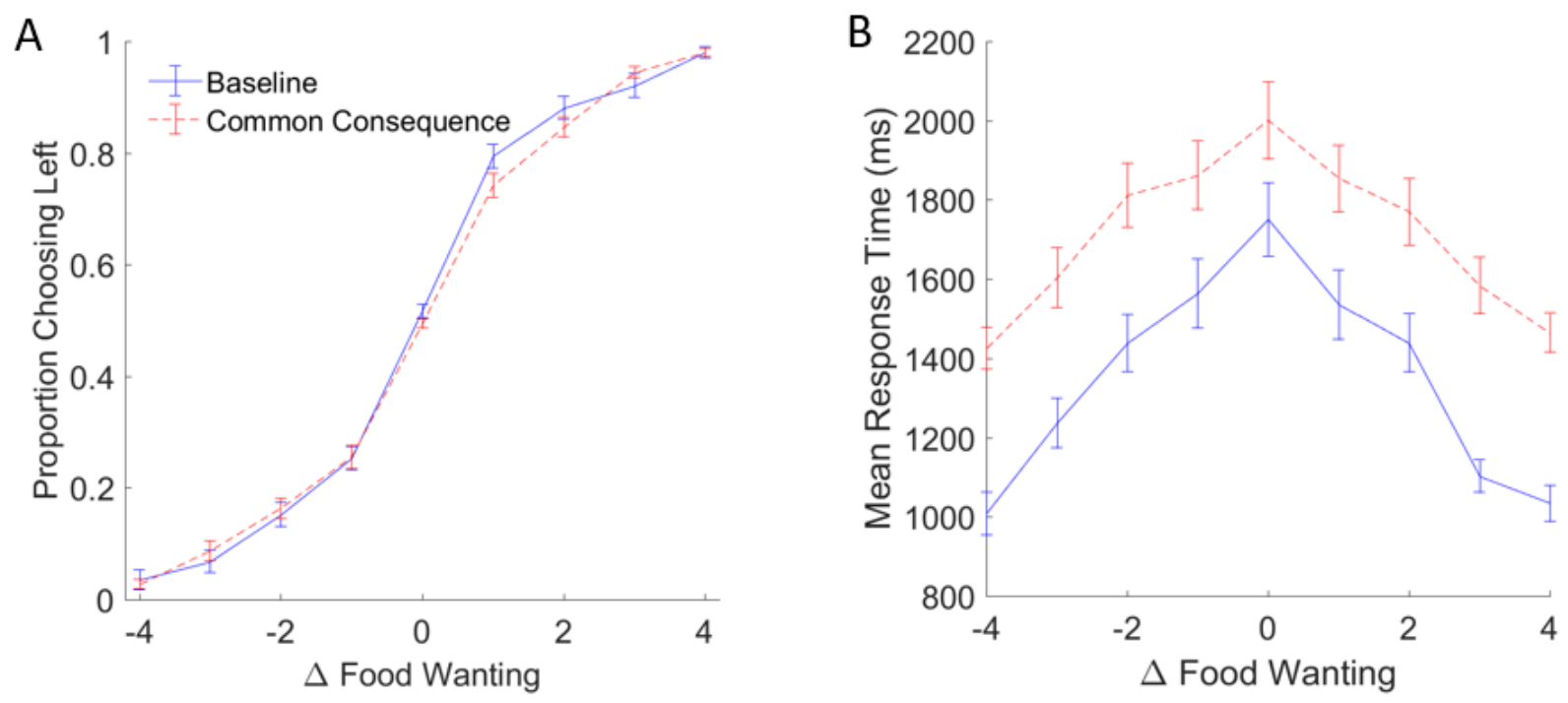

Fig. 2. The introduction of a common consequence alters psychometric properties of choice. Participants tended to choose the food option with a relatively greater subjective value as measured by differences (left - right) in wanting ratings (a). Mean response time (RT) was longest for trials on which the two foods were most closely matched in subjective value, and shortest for trials with large differences in subjective value (b). For both panels, error bars represent standard error of the mean computed across participants.

\section{Indulgent common consequences can encourage disciplined choices}

We next examined choice by focusing on Baseline image-image conflict trials, those involving a choice between one more-healthful, less-tasty option (i.e., a "disciplined" choice) and another less-healthful, more-tasty option (i.e., an "indulgent" choice). Since those trials involve a tradeoff between health and taste attributes, any change in the relative value participants place on one or both of those attributes could potentially alter choices. Participants chose the disciplined option in only $22 \%$ of all Baseline trials (dashed lines in Figure 3), reflecting the greater weight that taste information exerts on dietary choices. Disciplined choices were more frequent for Health Primed participants than for Taste Primed participants (Figure S1; 29\% vs. 17\%; 95\% CI $=[2.15,21.88], \mathrm{d}=0.58, \mathrm{t}(69)=2.43, \mathrm{p}=0.02)$, indicating that our prime altered choice behavior.

If the common consequence did not influence the processes of choice, then the proportion of healthy choices should be identical between the CI, CD, and Baseline tasks. Instead, we found that the introduction of an indulgent common consequence $(\mathrm{CI})$ roughly doubled the overall proportion of disciplined choices compared both to the Baseline task and to the CD trials (Figure 3; Figure S2). These effects replicated in both priming groups, both for the comparison between $\mathrm{CI}$ and baseline (all participants, $50 \%$ vs. $22 \%$; $95 \% \mathrm{CI}=[20.36,34.99], \mathrm{d}=1.29, \mathrm{t}(70)=7.54, \mathrm{p}$ $=1 \times 10^{-10}$; Health prime, $46 \%$ vs. $29 \% ; 95 \% \mathrm{CI}=[6.38,28.96], \mathrm{d}=0.74, \mathrm{t}(34)=3.18, \mathrm{p}=.003$; Taste prime, $54 \%$ vs. $\left.17 \% ; 95 \% \mathrm{CI}=[28.67,46.12], \mathrm{d}=2.12, \mathrm{t}(35)=8.70, \mathrm{p}=3 \times 10^{-10}\right)$ and for the comparison of CI and CD trials (all participants, $25 \%$ vs. 50\%; 95\% CI $=[-32.04,-18.58], \mathrm{d}$ 
$=-1.24, \mathrm{t}(70)=-7.50, \mathrm{p}=2 \times 10^{-10} ;$ Health prime, $25 \%$ vs. $46 \% ; 95 \% \mathrm{CI}=[-31.15,-11.25], \mathrm{d}=-$ $0.95, \mathrm{t}(34)=-4.33, \mathrm{p}=1 \times 10^{-4}$; Taste prime, $25 \%$ vs. $54 \% ; 95 \% \mathrm{CI}=[-38.69,-19.93], \mathrm{d}=-1.63$, $\left.\mathrm{t}(35)=-6.34, \mathrm{p}=3 \times 10^{-7}\right)$. These effects also replicated across individual participants (Figure S3) and held when including all CC and NCC trials in the analyses (Figure S4), which provides further support for their generality.

There was no difference in the proportion of disciplined choices between the Baseline and CD trials except in taste-primed participants (all participants, $25 \%$ vs. $22 \%$; 95\% CI $=[-2.64,7.36]$, $\mathrm{d}=0.12, \mathrm{t}(70)=0.94, \mathrm{p}=0.35$; Health prime, $25 \%$ vs. $29 \% ; 95 \% \mathrm{CI}=[-12.04,4.97], \mathrm{d}=-0.16$, $\mathrm{t}(34)=-0.84, \mathrm{p}=0.40$; Taste prime, $25 \%$ vs. $17 \% ; 95 \% \mathrm{CI}=[2.97,13.21], \mathrm{d}=0.46, \mathrm{t}(35)=$ $3.21, \mathrm{p}=.003)$.

These results show that the introduction of an indulgent common consequence dramatically increased the proportion of disciplined choices. We next explore one potential mechanism for these effects: value normalization.

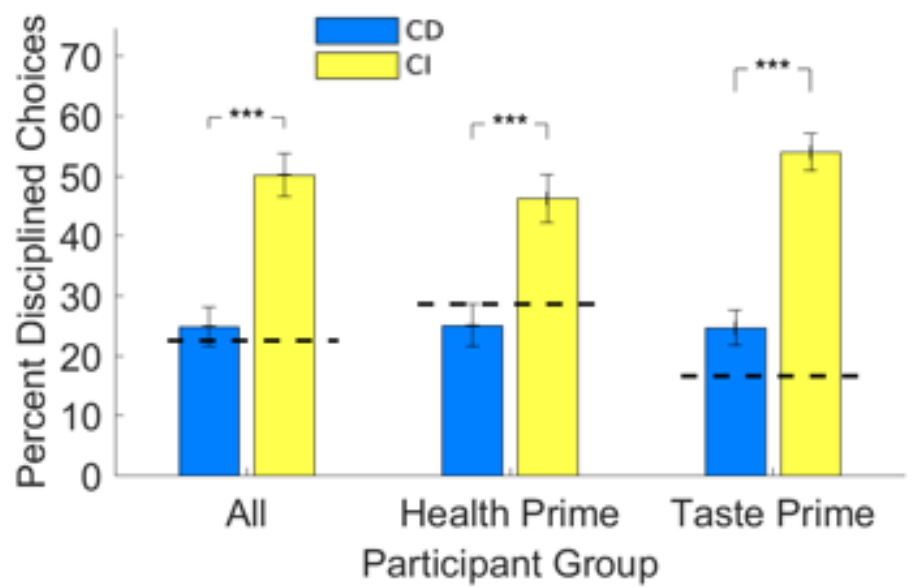

Fig. 3. Effect of common disciplined (CD) and common indulgent (CI) consequences on dietary choices. The introduction of a CI consequence led to significant and large-magnitude increases in the number of disciplined choices (plot a; i.e., choices of a more healthful but less tasty gamble). The dashed lines show the proportion of disciplined choices in the Baseline task for each group of participants. Error bars represent standard error of the mean computed across participants. Significance indicators of $* * *$, and $* * *$ represent $\mathrm{p}<.05, .01$, and .001 respectively.

\section{Contextual effects on taste and health decision weights: Simulated evidence}

Context effects have profound effects on choice behavior; like other psychological continua, subjective value is normalized in comparison to contextual stimuli by physiological processes within the brain (Carandini \& Heeger, 2011; Louie, Khaw, \& Glimcher, 2013). Here, we use simulated data to illustrate how a value normalization process could partially account for our observed common consequence effects. 
Consider the Baseline choice between a unique disciplined food $(\mathrm{x})$ and a unique indulgent food (y), each with $i$ attributes (here, health and taste). Under a standard value normalization model, the value associated with each attribute $i$ depends on the range of that attribute within the set (Equation 1).

$$
v\left(x_{i} ; y_{i}\right)=\frac{x_{i}}{x_{i}+y_{i}}
$$

A third option $\left(\mathrm{z}_{i}\right)$ changes the values over which each attribute is normalized according to Equation 2 (Landry \& Webb, 2018). This assumes an initial attribute-wise normalization, before normalizing across options.

$$
v\left(x_{i} ; y_{i}, z_{i}\right)=\frac{x_{i}}{x_{i}+y_{i}}+\frac{x_{i}}{x_{i}+z_{i}}
$$

The ratio of equations 1 and 2 represents the relative influence of option $\mathrm{z}$ on normalized value (Equation 3; Landry and Webb, 2018).

$$
\beta_{i}=\frac{v\left(x_{i} ; y_{i}, z_{i}\right)}{v\left(x_{i} ; y_{i}\right)}
$$

We propose that common consequences, despite technically being irrelevant to the choice process, altered decision weights through a process of value normalization. Under this proposal, the CD condition normalized over a larger range of values for the health attribute, while the CI condition normalized over a larger range of values for the taste attribute. We illustrated these effects using a simulation of choices between a fixed unique disciplined and a unique indulgent food when a common consequence, $\mathrm{z}$, is added to the choice set. Each trial in the simulated choice set had the same unique indulgent and disciplined options - and $\mathrm{z}$ ranged from neutral to extremely disciplined or indulgent (see Figure S5a for the simulation stimulus set). Next, we calculated the normalized taste and health values for each unique food (see Figure S5b) using equations (1) and (2). Then, we calculated the simulated weight $\beta_{i}$ for both taste and health using equation (3).

The results (Figure S5c) indicated that common consequences alter the subjective decision weights of the unique foods. Specifically, as a common indulgent item increased in tastiness and decreases in healthfulness (cool colors in Figure S5c), decision weights for taste decreased but decision weights for health increased; this pattern would potentiate increasingly disciplined choices. The opposite effect was observed as the common consequence became more disciplined: increased decision weights for taste but diminished decision weights for health, which would potentiate indulgent choices.

We next assessed whether the difference in normalized values between CI and CD conditions predicted our observed increase in disciplined choices in the CI condition (eq. 2), even when controlling for individual preferences (See Supplemental Methods for details). To do this on a participant-by-participant basis, we simulated 100 trials between pairs of foods whose taste and health values were normalized by the weights that participant placed on taste and health in the Baseline task. The proportion of disciplined choices was then calculated using a softmax function and a temperature parameter $\sigma$ estimated from the Baseline task (eq. 4). We found that the number of disciplined choices predicted in the CI condition was greater relative to $\mathrm{CD}$ condition (Fig. S6; mean proportion increase $=0.03 ; \mathrm{d}=0.45, \mathrm{t}(70)=3.80,95 \% \mathrm{CI}=[0.01$ 0.05], $\mathrm{p}=0.0003$ ). Moreover, the predicted shift in disciplined choices from the Baseline task to $\mathrm{CC}$ conditions was greater in the $\mathrm{CI}$ than $\mathrm{CD}$ condition, similar to what was observed in our 
actual data (means $=-0.01,0.02 ; \mathrm{d}=-0.27, \mathrm{t}(78)=-3.10,95 \% \mathrm{CI}=[-0.04-0.01], \mathrm{p}=0.003)$. Together, these simulated results suggested one mechanism through which indulgent CCs could induce more disciplined choices, while disciplined CCs induce more indulgent choices. Of note, normalization does not fully account for the observed shift in behavior. This indicates that multiple mechanisms are likely at work to exhibit the shift in disciplined choice in the CI condition - a possibility we consider in our later analyses of eye gaze measures.

$$
p\left(x_{i}\right)=\frac{e^{\frac{v\left(x_{i} ; y_{i}, z_{i}\right)}{\sigma}}}{e^{\frac{v\left(x_{i} ; y_{i}, z_{i}\right)}{\sigma}}+e^{\frac{v\left(x_{j} ; y_{j}, z_{j}\right)}{\sigma}}}
$$

We next evaluated whether the incorporation of value normalization - using the procedures in this simulation - improves predictions of choice behavior in our experiment. We compared two models: one that uses only raw participant ratings for the unique stimuli, the other that uses participant ratings that have been adjusted by the value normalization process. In both cases, each attribute's values were normalized by their weights in the Baseline task, estimated using mixed-effect logistic regression, to account for differences in underlying preference for each attribute. Both models were estimated using mixed-effects logistic regression with random slopes and intercept, and model comparison used maximum likelihood estimation (See Supplemental Methods for estimation details). We found that our data were better fit by the normalization model than when using weighted ratings alone. For CD trials, the normalization model was superior both when considering the entire subject sample (mean normalization BIC $=207$, nonnormalization $\mathrm{BIC}=448$ ) and when considering each subject individually (i.e., it was a better fit for 68 of 73 participants). For CI trials, the normalization model was again a better fit over the entire sample (mean normalization $\mathrm{BIC}=69$, non-normalization $\mathrm{BIC}=164$ ) and when considering individual participants (i.e., it was better for 69 out of 73 of participants). Further, normalized value model predicts more choices correctly in the $\mathrm{CI}$ than $\mathrm{CD}$ condition $(95 \% \mathrm{CI}=$ $\left.[58.68,79.98], \mathrm{d}=-3.06, \mathrm{t}(70)=-25.81, \mathrm{p}<1 \times 10^{-10}\right)$.

Both our simulations and model comparison results support the conclusion that the observed changes in choice behavior associated with the introduction of a common consequence are consistent with the outcome of a value normalization process.

\section{Attribute decision weights and common consequence: Experimental evidence}

Our simulation predicted that the common consequences alter how individuals use health and taste information to guide their choices. To confirm this prediction, we used logistic regressions to determine the decision weights associated with relative taste and relative health, as independent predictors of choices. We then compared these decision weights between the Baseline and Common Consequence tasks. To account for possible differences in the range of food ratings between the two tasks, we normalized value differences for each task from -1 to 1 .

The attributes of the common consequence led to dramatic changes in decision weights. Taste weights were smaller, and health weights were less negative, in the CI condition compared to the CD condition, for both prime groups (taste decision weights, all participants: $95 \% \mathrm{CI}=[-0.79$, $0.26], \mathrm{d}=-0.24, \mathrm{t}(70)=-4.01, \mathrm{p}=1 \times 10^{-4}$; Health Prime, 95\% CI $=[-0.92,-0.15], \mathrm{d}=-0.27, \mathrm{t}(34)$ 
$=-2.83, \mathrm{p}=.008$; Taste Prime, 95\% CI $=[-0.89,-0.14], \mathrm{d}=-0.28, \mathrm{t}(35)=-2.80, \mathrm{p}=.008$; health decision weights, all participants: $95 \% \mathrm{CI}=[0.24,0.78], \mathrm{d}=0.23, \mathrm{t}(70)=3.73, \mathrm{p}=4 \times 10^{-4}$; Health Prime, $95 \% \mathrm{CI}=[0.14,0.96], \mathrm{d}=0.23, \mathrm{t}(34)=2.75, \mathrm{p}=.009$; Taste Prime, $95 \% \mathrm{CI}=$ $[0.08,0.85], \mathrm{d}=0.23, \mathrm{t}(35)=2.48, \mathrm{p}=.02)$. These results suggest that the less negative health, and less positive taste, decision weights closer to zero in the CI condition, compared to CD condition, for both primes may help facilitate healthier choices in that condition. This result confirms the qualitative predictions from our simulations, and points to a mechanism through which the CI condition could facilitate disciplined choices.

Next, we compared taste and health decision weights in the Common Consequence conditions to those in the Baseline task (dotted lines in Figure 4). The weight on health became less negative from the Baseline task to the CI condition. The weight on health became more negative from the Baseline task to the CD condition for the Taste, but not Health, primed participants (CI condition: all participants, 95\% CI $=[1.64,3.12], \mathrm{d}=0.87, \mathrm{t}(70)=6.40, \mathrm{p}=2 \times 10^{-8}$; Health Prime, $95 \% \mathrm{CI}=[0.19,2.42], \mathrm{d}=0.46, \mathrm{t}(34)=2.37, \mathrm{p}=.02$; Taste Prime, $95 \% \mathrm{CI}=[2.53,4.32]$, $\mathrm{d}=1.39, \mathrm{t}(35)=7.73, \mathrm{p}=4 \times 10^{-9} ; \mathrm{CD}$ condition: all participants, 95\% $\mathrm{CI}=[1.12,2.62], \mathrm{d}=0.64$, $\mathrm{t}(70)=5.00, \mathrm{p}=4 \times 10^{-6}$; Health Prime, 95\% CI $=[-0.30,1.81], \mathrm{d}=0.25, \mathrm{t}(34)=1.46, \mathrm{p}=.15$; Taste Prime, $\left.95 \% \mathrm{CI}=[1.98,3.93], \mathrm{d}=1.10, \mathrm{t}(35)=6.16, \mathrm{p}=5 \times 10^{-7}\right)$. Although there were no overall differences in taste weights from the Baseline to $\mathrm{CI}$ condition $(95 \% \mathrm{CI}=[-0.86,0.35], \mathrm{d}$ $=-0.10, \mathrm{t}(70)=-0.85, \mathrm{p}=0.40)$, we find that there was for the Taste, but not Health, Primed participants (Health prime, 95\% CI $=[-0.63,1.36], \mathrm{d}=0.13, \mathrm{t}(34)=0.75, \mathrm{p}=0.46$; Taste Prime, $95 \% \mathrm{CI}=[-1.55,-0.18], \mathrm{d}=-0.38, \mathrm{t}(35)=-2.56, \mathrm{p}=0.02)$. There were no differences in taste weights from the Baseline to CD condition (all participants, 95\% $\mathrm{CI}=[-0.34,0.87], \mathrm{d}=0.10$, $\mathrm{t}(70)=0.88, \mathrm{p}=0.38$; Health Prime, 95\% CI $=[-0.10,1.91], \mathrm{d}=0.31, \mathrm{t}(34)=1.83, \mathrm{p}=0.08$; Taste Prime, $95 \% \mathrm{CI}=[-1.03,0.33], \mathrm{d}=-0.15, \mathrm{t}(35)=-1.05, \mathrm{p}=0.30)$. See Figure $\mathrm{S} 7$ for these weights plotted relative to the Baseline task, and Figure S8 for these weights after normalizing taste and health ratings across all three conditions. Overall, the above results reflect the large influence of the prime on the Baseline task (see Fig. S1), and overall lack of the prime's influence in the Common Consequence task.

Together, these changes in decision weights provide additional experimental evidence to support our simulated results; an indulgent common consequence paradoxically increases the weight placed on health, relative to taste, in choice compared to a disciplined common consequence. 

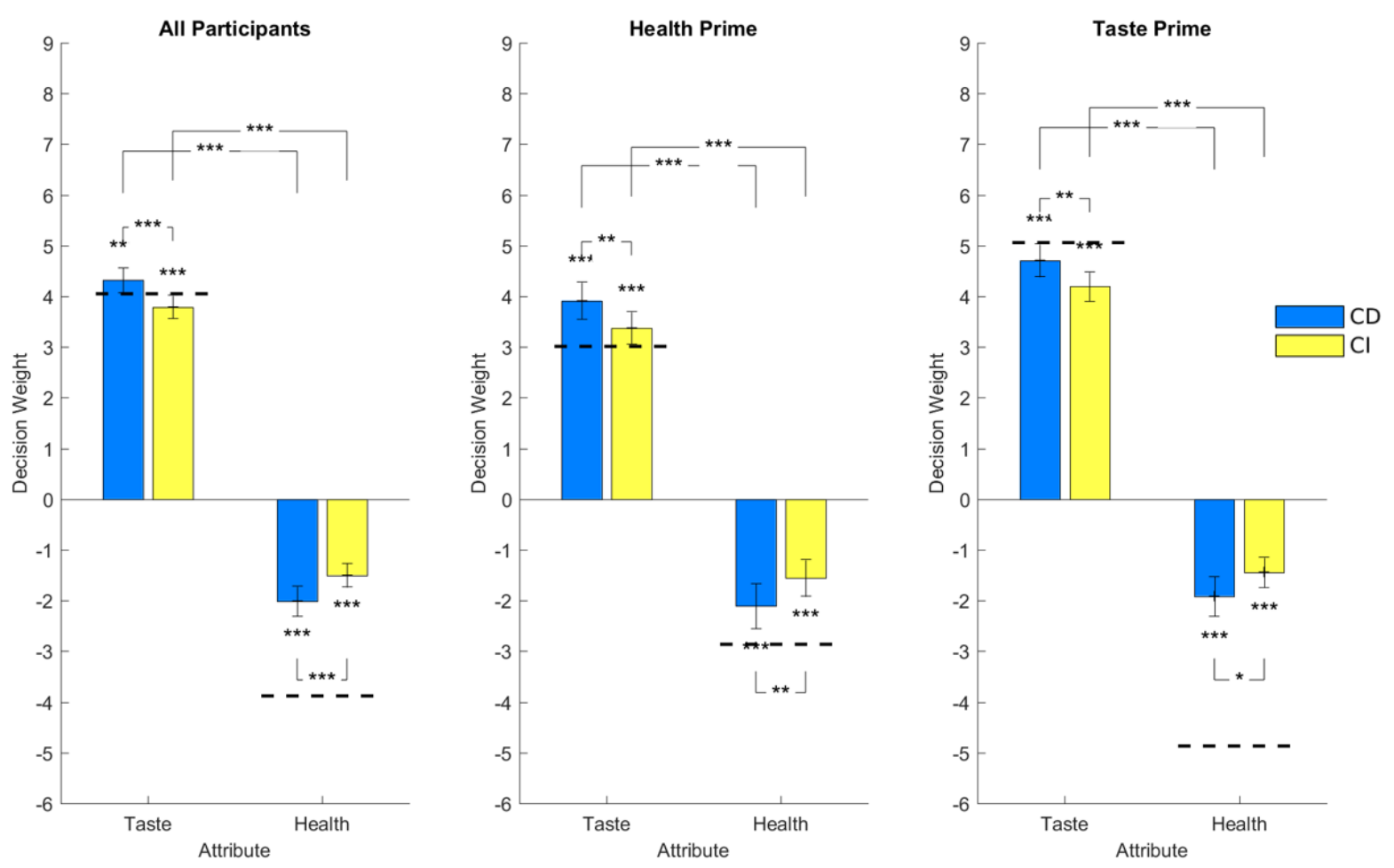

Fig. 4. Decision weights by Task and CC condition. The bar graph plots the influence of taste and health information, as estimated by logistic decision weights, upon decisions involving common disciplined (CD) or indulgent (CI) common consequences. The dashed lines represent decision weights in the Baseline task for each priming group. For all panels, error bars represent standard error of the mean computed across participants. Significance indicators of *, **, and $* * *$ represent $\mathrm{p}<.05, .01$, and .001 respectively.

\section{Increased gaze to healthful foods in Common Indulgent condition}

Next, we used eye-tracking methods to evaluate whether common consequences drive attention toward salient outlier foods. First, we verified that participants' eye gaze followed expected patterns for both the Baseline and Common Consequence tasks. There were more fixations to each food when the choice was difficult, relative to when one option was clearly favored, in both tasks (Figure 5a; quadratic slope, Baseline all participants, slope $=-1.02,95 \% \mathrm{CI}=[-1.28$, $0.76], \mathrm{d}=-2.05, \mathrm{t}(19729)=575.28, \mathrm{p}<1 \times 10^{-10}$; Health prime, slope $=-1.07,95 \% \mathrm{CI}=[-1.45,-$ $0.69], \mathrm{d}=-2.04, \mathrm{t}(9812)=285.66, \mathrm{p}<1 \times 10^{-10}$; Taste prime, slope $=-0.96,95 \% \mathrm{CI}=[-1.32$, $0.60], \mathrm{d}=-2.05, \mathrm{t}(9912)=297.36, \mathrm{p}<1 \times 10^{-10}$; CC quadratic slope: all participants, slope $=-$ $1.42,95 \% \mathrm{CI}=[-1.76,-1.09], \mathrm{d}=-3.80, \mathrm{t}(14941)=423.06, \mathrm{p}<1 \times 10^{-10}$; Health prime, slope $=-$ $1.36,95 \% \mathrm{CI}=[-1.79,-0.93], \mathrm{d}=-6.95, \mathrm{t}(7388)=267.63, \mathrm{p}<1 \times 10^{-10}$; Taste prime, slope $=-$ $\left.1.50,95 \% \mathrm{CI}=[-2.01,-0.98], \mathrm{d}=-2.69, \mathrm{t}(7548)=173.82, \mathrm{p}<1 \times 10^{-10}\right)$. As with choice and reaction time data previously reported (Fig 2), the number of eye fixations was more tightly linked to food wanting value in the Common Consequence task, assessed by larger quadratic slope coefficients in the Common Consequences task (all participants, 95\% $\mathrm{CI}=[0.29,0.53], \mathrm{d}=$ 
$0.92, \mathrm{t}(70)=6.71, \mathrm{p}=4 \times 10^{-9}$; Health prime, $95 \% \mathrm{CI}=[0.20,0.54], \mathrm{d}=0.85, \mathrm{t}(34)=4.44, \mathrm{p}=$ $9 \times 19^{-5}$; Taste prime, $\left.95 \% \mathrm{CI}=[0.26,0.62], \mathrm{d}=0.99, \mathrm{t}(35)=4.99, \mathrm{p}=2 \times 10^{-5}\right)$.
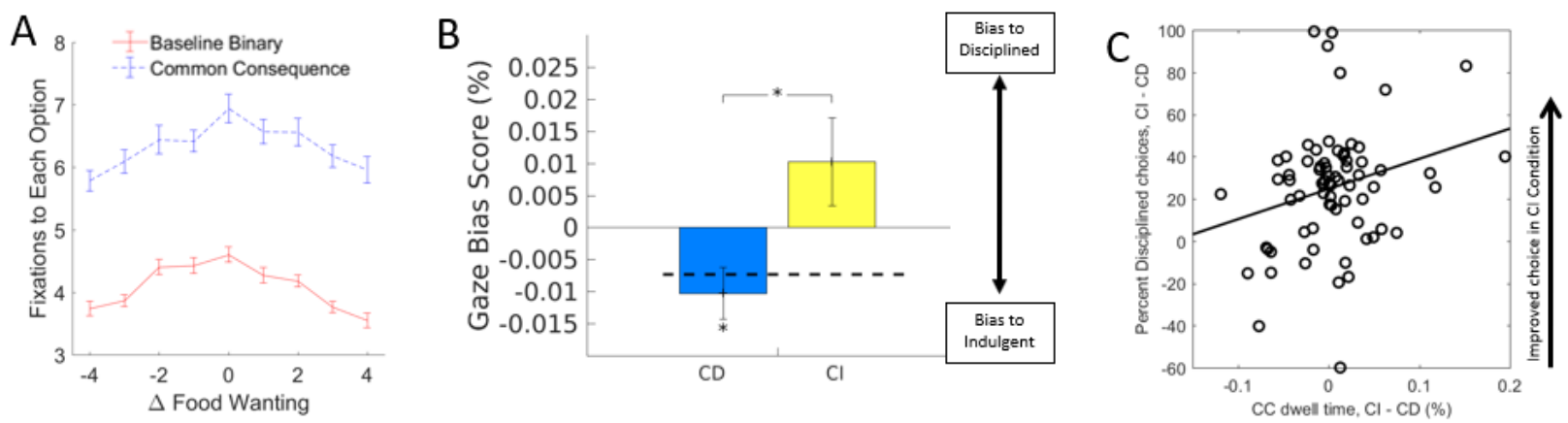

Fig. 5. Results of the gaze bias analysis. Average fixations to each option in the Baseline and Common Consequence tasks are shown in (a). In bar graph (b), gaze bias scores are graphed, and gaze bias for the Baseline task is represented by a black dashed line. Positive scores represent more fixations to the disciplined unique option, and negative numbers represent more fixations to the indulgent unique option. Error bars in plots a-b represent standard error computed across subjects. Plot (c) demonstrates that the relative proportion of total dwell time spent fixating on the Common Consequence in the CI vs. CD conditions is positively correlated with disciplined choices. For a-b, error bars represent standard error of the mean computed across participants. For b, significance indicators of $*$ and $* *$ represent $\mathrm{p}<.05$ and .01 , respectively.

For each participant, we next calculated a gaze bias score that represented the difference in gaze dwell time between the disciplined and indulgent unique foods in the Common Consequences task (see Methods). A positive gaze bias score reflected longer relative gaze to the disciplined unique food, and a negative score reflected increased gaze to the indulgent unique food. Across all participants, gaze was biased slightly toward the indulgent unique item in CD trials (Figure $5 b, 95 \% \mathrm{CI}=[-0.02,-0.00], \mathrm{d}=-0.30, \mathrm{t}(70)=-2.54, \mathrm{p}=0.01)$, which was not different from the gaze bias seen in the Baseline task (dotted line, Figure 5b, 95\% CI $=[-0.01,0.02], d=0.07, t(70)$ $=0.37, \mathrm{p}=0.71)$. Moreover, there was a greater gaze bias toward the disciplined item the CI condition, where there were more disciplined choices, relative to the $\mathrm{CD}$ condition $(95 \% \mathrm{CI}=$ $[0.00,0.04], \mathrm{d}=0.43, \mathrm{t}(70)=2.64, \mathrm{p}=0.01)$. In addition, there was a larger bias toward the disciplined unique option in the CI condition compared to the Baseline task $(95 \% \mathrm{CI}=[-0.03$, $0.00], \mathrm{d}=-0.32, \mathrm{t}(70)=-2.05, \mathrm{p}=0.04)$. These results indicated that the identity of the common consequence influenced eye gaze to the unique food items. Specifically, in both the Baseline task and CD trials, gaze was biased in the same way - toward the unique indulgent food. However, the introduction of a common indulgent consequence shifted this bias away from the indulgent food, and toward the disciplined unique food instead. This could explain not only the behavioral shift between CI and CD conditions, but also why there is no large behavioral difference between $\mathrm{CD}$ and baseline trials; the disciplined common consequence was unable to draw attention away from the baseline fixation pattern, which was already biased toward indulgent item(s). 
Converging evidence from both simulated and experimental results above suggests that the attributes of the common consequence can dramatically bias choice. Previous research has found that the value of the attended item is enhanced in the value comparison process (Krajbich, Armel, \& Rangel, 2010). If this is the case, increased attention to the CC could increase the weight of its attributes during attribute normalization, further biasing the value of the unique foods. We investigated this in our data by looking at the influence of attention (as estimated by eye gaze) to the common consequence on the across-condition behavioral bias. That is, we estimated a linear regression predicting the proportion healthy choices in the $\mathrm{CI}$, relative to $\mathrm{CD}$, condition based on attentional bias to the $\mathrm{CC}$ in each condition. We find that greater attention to the common consequence in the CI, relative to $\mathrm{CD}$, condition is associated with increased decision bias (Fig. 5c; slope $=143.21,95 \% \mathrm{CI}=[14.27,272.15], \mathrm{r}=0.26, \mathrm{~F}(69)=4.91, \mathrm{p}=$ 0.03 ). To ensure that this effect is not driven by outliers, we also ran a robust regression (slope $=$ $134.30,95 \% \mathrm{CI}=[19.76,248.84], \mathrm{t}(69)=2.34, \mathrm{p}=0.02)$. This indicates that increased gaze to the irrelevant common consequence between conditions enhanced the behavioral bias between conditions. This suggests a mechanism for susceptibility to context effects: the more strongly one's gaze is drawn to the irrelevant option, the more one is biased by the identity of that option. 


\section{Discussion}

Outside the laboratory, efforts to introduce more healthy food options in grocery stores have largely not been able to improve choices and health outcomes (Cummins et al., 2014; e.g., Elbel et al., 2015; e.g., Morland et al., 2002; Seymour et al., 2004). Because such interventions necessarily change the array of available foods, the associated context effects may have unintended negative consequences. In this study, we describe the mechanisms by which the introduction of new foods - here, irrelevant common consequences that should not affect preferences between unique choice options - can have powerful influences on choice. We found, paradoxically, that disciplined choices are potentiated not by the availability of disciplined foods, but rather by the introduction of an indulgent food to the choice set. Of note, we found that the introduction of a disciplined food neither improved nor worsened choice, reflecting the fact that participants already attend to indulgent foods by default (cf., data from the Baseline task). Using theories of how the brain adjusts value information according to its context (e.g., Carandini \& Heeger, 2011), we showed how indulgent foods may alter the perceived weights ascribed to taste and health information. We found that indulgent foods alter attention as measured by eye gaze, and drastically increase the proportion of healthy choices. Moreover, we found that participants who showed the greatest attentional biases also showed the greatest choice biases, suggesting why some individuals are more swayed by choice context than others.

Our results build upon a rich literature on how choices are susceptible to contextual influences (e.g., Payne, 1982; Prelec et al., 1997; Rooderkerk et al., 2011). For example, previous work has found that "phantom alternatives" - options shown but currently unavailable for choice - can bias behavior through context effects (Doyle, O'Connor, Reynolds, \& Bottomley, 1999; Karmarkar, 2017; Pratkanis \& Farquhar, 1992; Scarpi \& Pizzi, 2013; Trueblood \& Pettibone, 2017). The current paper provides four extensions of this prior work.

First, this study suggests an avenue through which dietary choice can be improved without relying on an individual's ability to exert self-control. That is, we find little evidence that participants' selection of the healthier option in trials with more indulgent foods was facilitated by momentary improvements in willpower. Given that dietary self-control is both difficult to deploy and difficult to improve (Marketdata Enterprises Inc., 2014; Paeratakul, York-Crowe, Williamson, Ryan, \& Bray, 2002), and that that changes in choice architecture can be more effective than attempts to change the decision maker (Johnson et al., 2012), future interventions that focus on choice sets may be particularly promising.

Second, we demonstrate that normalization, the process by which neurons encode information about stimulus magnitude (Carandini \& Heeger, 2011; Louie et al., 2013), can partially explain the paradoxical behavioral effects we observe. Normalization is grounded in models of how neural circuits function, and is a fundamental property of psychological quantities. This phenomenon has been observed in natural judgments and decisions, and can explain phenomena such as range effects (Soltani, De Martino, \& Camerer, 2012). In dietary choice, we propose that a food's tastiness and healthfulness will be encoded and perceived not in absolute terms, but instead relative to the other options in a choice set. Our simulations show how both the tastiness and healthfulness of a disciplined food - that is, one that is objectively healthy but not tasty may be enhanced by the introduction of an additional indulgent food. This altered perception then may partially explain the increased number of healthy choices we observe. These simulations provided falsifiable predictions that we then, in turn, partially confirmed in the 
experimental data. Although we do not have conclusive evidence as to why adding a common disciplined item does not markedly worsen choice, as predicted by the normalization theory, we use evidence from the eye tracking data to propose a hypothesis. Because gaze was not more biased toward the unhealthy item in the Common Disciplined condition than in the Baseline task, attention remained directed to the unique indulgent food in both cases - leading to a similar pattern of indulgent choices.

Third, we present a novel choice paradigm that introduces a probabilistic dietary choice task. This task allowed us to test the influence of a food without changing the core feature of the task, which should involve choices over the items unique to the options - and should not be influenced by the common consequence. However, this task still is fully incentive-compatible, since any of the foods could be selected for later consumption. Real world extensions of this task could include restaurants offering the chance of a sweet option to encourage more healthy choices.

Fourth, we took advantage of real-time estimates of attentional processes from eye tracking to provide a mechanistic explanation for these context effects. Such insights are important for understanding how these context effects work to bias the decision process, and for suggesting potential behavioral interventions. For example, if a context effect results from changes in the allocation of attention - as observed in this study - then it may be mitigated or enhanced by manipulations that diminish or enhance this bias, such as by making healthier items more prominent or by decreasing the visual salience of tempting indulgent items (Bordalo, Gennaioli, \& Shleifer, 2013). In addition, through training and education, individuals could learn to avoid focusing on irrelevant contextual cues such as healthy but aversive items, which are irrelevant because they would never be chosen by the consumer.

Our sample included participants as young as 18 and old as 60 and drew participants from both the university and wider community. However, the majority of the participants in our sample were young adults, with an average age of 24 years old. As with all research using this type of convenience sample, there are significant constraints on the generalizability of our findings. Our sample is drawn from an industrialized, developed nation, for which issues related to dietary self control may be very different from that of other parts of the world where food insecurity is more common (Henrich et al., 2012). Research has suggested that demographic information, such as country of origin, can influence - but not remove - context effects like those reported here (e.g. Kim et al. 2016). Further, this study was conducted in a controlled, laboratory setting, which limits our ability to draw conclusions about effects in real-world settings (List and Levitt, 2007).

It is also important to note that our study cannot disambiguate between two competing mechanisms that may drive our behavioral effect. One possibility is that context may influence an underlying value mechanism, perhaps via normalization, to bias choice that is then reflected in changes in eye gaze (Krajbich et al., 2010). Alternatively, context may influence attention, which is reflected in eye gaze and thereby influences the choice process to bias choice. Given the interrelations between value and attention found in our results, we speculate that both effects will be elicited in many choices - leading to a positive feedback loop where value drives attention to particular options, which in turn increases those options' subjective value. Future studies that manipulate attention (e.g., by forcing gaze at the indulgent or disciplined options) could help disentangle the directionality of this effect.

Additionally, our results indicate that individual variation in the decision process, as estimated through our eye gaze measure, was reflected in individual differences in sensitivity to contextual 
influences. This suggests that some individuals may be more or less susceptible to contextual effects in real-world choices. For example, the ability to exert financial self-control, such as saving for retirement rather than splurging on luxury goods today, could be linked to attentional effects driven by the choice context. Or, risk seeking behavior among those susceptible to excessive drug use, alcohol consumption, or gambling could be shifted by altering the context in which these choices are often made. An improved understanding of the connections between context, attention, and choice will help target interventions toward individuals most susceptible to their influence - potentially increasing the likelihood of disciplined choices. 


\section{Author Contributions}

All authors participated in designing the study. N. Sullivan collected and analyzed the data, with input from S. Huettel. N. Sullivan and S. Huettel wrote the paper, with contributions from all authors.

\section{Declaration of Conflicting Interests}

The authors declared that they had no conflicts of interest with respect to their authorship or the publication of this article.

\section{Acknowledgments}

We thank Ryan Webb and Peter Landry for insights on value normalization derived from their ongoing work. We thank Jonathan Winkle for creating the photographs used in this study. We thank Christopher Ong and Indy Rajan for assistance with data collection. This project was supported by institutional funding from Duke University. 


\section{References}

Allais, M. (1953). Le Comportement de l'Homme Rationnel devant le Risque: Critique des Postulats et Axiomes de l'Ecole Americaine. Econometrica, 21(4), 503-546.

Bordalo, P., Gennaioli, N., \& Shleifer, A. (2013). Salience and Consumer Choice. Journal of Political Economy, 121(5), 803-843.

Brainard, D. H. (1997). The Psychophysics Toolbox. Spatial Vision, 10(4), 433-436.

Carandini, M., \& Heeger, D. J. (2011). Normalization as a canonical neural computation. Nature reviews. Neuroscience, 13(1), 51-62.

Chakravarti, D. (1983). A framework for exploring context effects on consumer judgment and choice. ACR North American Advances.

Cummins, S., Flint, E., \& Matthews, S. A. (2014). New neighborhood grocery store increased awareness of food access but did not alter dietary habits or obesity. Health Affairs, 33(2), 283-291.

Deng, T. (2009). McDonald's New Communication Strategy on Changing Attitudes and Lifestyle. International Journal of Marketing Studies, 1(1), 37.

Doyle, J. R., O'Connor, D. J., Reynolds, G. M., \& Bottomley, P. A. (1999). The robustness of the asymmetrically dominated effect: Buying frames, phantom alternatives, and in-store purchases. Psychology and Marketing, 16(3), 225-243.

Dumanovsky, T., Huang, C. Y., Nonas, C. A., Matte, T. D., Bassett, M. T., \& Silver, L. D. (2011). Changes in energy content of lunchtime purchases from fast food restaurants after introduction of calorie labelling: cross sectional customer surveys. The BMJ, 343, d4464.

Elbel, B., Moran, A., Dixon, L. B., Kiszko, K., Cantor, J., Abrams, C., et al. (2015). Assessment of a government-subsidized supermarket in a high-need area on household food availability and children's dietary intakes. Public Health Nutrition, 18(15), 2881-2890.

Huber, J., Payne, J. W., \& Puto, C. (1982). Adding Asymmetrically Dominated Alternatives: Violations of Regularity and the Similarity Hypothesis. Journal of Consumer Research, 9(1), 90-98.

Jargon, J. (2013, October 18, 2013). At McDonald's, salads just don't sell. Wall Street Journal. Retrieved April 28, 2017,

Johnson, E. J., Shu, S. B., Dellaert, B. G. C., Fox, C., Goldstein, D. G., Häubl, G., et al. (2012). Beyond nudges: Tools of a choice architecture. Marketing Letters, 23(2), 487-504.

Kahneman, D., \& Tversky, A. (1979). Prospect Theory: An Analysis of Decision under Risk. Econometrica, 47(2), 263-291.

Karmarkar, U. R. (2017). The Impact of "Display-Set" Options on Decision-Making. Journal of Behavioral Decision Making, 30(3), 744-753.

Krajbich, I., Armel, C., \& Rangel, A. (2010). Visual fixations and the computation and comparison of value in simple choice. Nature Neuroscience, 13(10), 1292-1298.

Landry, P., \& Webb, R. (2018). Pairwise Normalization: A Neuroeconomic Theory of Multi-Attribute Choice. Social Science Research Network.

Louie, K., Khaw, M. W., \& Glimcher, P. W. (2013). Normalization is a general neural mechanism for context-dependent decision making. Proceedings of the National Academy of Sciences, 110(15), 6139-6144.

Marketdata Enterprises Inc. (2014). The U.S. Weight Loss Market: 2014 Status Report \& Forecast.

Morland, K., Wing, S., \& Diez Roux, A. (2002). The contextual effect of the local food environment on residents' diets: the atherosclerosis risk in communities study. American Journal of Public Health, 92(11), 1761-1767.

Paeratakul, S., York-Crowe, E. E., Williamson, D. A., Ryan, D. H., \& Bray, G. A. (2002). Americans on Diet. Journal of the American Dietetic Association, 102(9), 1247-1251.

Payne, J. W. (1982). Contingent decision behavior. Psychological Bulletin, 92(2), 382. 
Pratkanis, A. R., \& Farquhar, P. H. (1992). A Brief History of Research on Phantom Alternatives: Evidence for Seven Empirical Generalizations About Phantoms. Basic and Applied Social Psychology, 13(1), 103-122.

Prelec, D., Wernerfelt, B., \& Zettelmeyer, F. (1997). The role of inference in context effects: Inferring what you want from what is available. Journal of Consumer Research, 24(1), 118-125.

Rooderkerk, R. P., Van Heerde, H. J., \& Bijmolt, T. H. (2011). Incorporating context effects into a choice model. Journal of Marketing Research, 48(4), 767-780.

Rosenheck, R. (2008). Fast food consumption and increased caloric intake: a systematic review of a trajectory towards weight gain and obesity risk. Obesity Reviews, 9(6), 535-547.

Savage, L. J. (1954). The foundations of statistics. New York: John Wiley.

Scarpi, D., \& Pizzi, G. (2013). The Impact of Phantom Decoys on Choices and Perceptions. Journal of Behavioral Decision Making, 26(5), 451-461.

Seymour, J. D., Yaroch, A. L., Serdula, M., Blanck, H. M., \& Khan, L. K. (2004). Impact of nutrition environmental interventions on point-of-purchase behavior in adults: a review. Preventive Medicine, 39 Suppl 2, S108-136.

Soltani, A., De Martino, B., \& Camerer, C. (2012). A range-normalization model of context-dependent choice: a new model and evidence. PLoS Computational Biology, 8(7), e1002607.

Sorensen, L. B., Moller, P., Flint, A., Martens, M., \& Raben, A. (2003). Effect of sensory perception of foods on appetite and food intake: a review of studies on humans. Int J Obes Relat Metab Disord, 27(10), 1152-1166.

Starmer, C. (1992). Testing New Theories of Choice under Uncertainty using the Common Consequence Effect. Review of Economic Studies, 59(4), 813-830.

Trueblood, J. S., Brown, S. D., Heathcote, A., \& Busemeyer, J. R. (2013). Not just for consumers: context effects are fundamental to decision making. Psychological Science, 24(6), 901-908.

Trueblood, J. S., \& Pettibone, J. C. (2017). The Phantom Decoy Effect in Perceptual Decision Making. Journal of Behavioral Decision Making, 30(2), 157-167.

Wilcox, K., Vallen, B., Block, L., \& Fitzsimons, G. J. (2009). Vicarious Goal Fulfillment: When the Mere Presence of a Healthy Option Leads to an Ironically Indulgent Decision. Journal of Consumer Research, 36(3), 380-393.

World Health Organization. (2016). Obesity and overweight fact sheet. 


\section{Supplemental Methods}

\section{Prime text}

\section{Health Prime}

The purpose of this study is to learn about how a food item's healthfulness affects people's choices about what they eat. We are interested in this question because leading scientists at top universities across the country have shown that eating a healthy diet is very important. They mention that one key benefit of eating healthy is the ability to maintain a healthy body weight, which can reduce the risk for many diseases. Previous research found that the top three killers in America are heart disease, cancer and stroke. Chronic diseases develop over time and are the cumulative effect of each eating decision we make in our lives. The health benefits of eating healthy are clear, but we would like to better understand how people incorporate health into each individual food choice.

\section{Taste Prime}

The purpose of this study is to learn how taste affects people's choices about the foods they eat. We are interested in this question because food is a central part of human culture, and is thought to be a source of enjoyment, passion, and fulfillment for many. Leading scientists at top universities across the country have found that high-taste foods reliably increase activity in the brain's reward centers. This increased activity is usually associated with a boost in dopamine levels in the brain, and dopamine is closely tied to our brain's reward processing, as well as our subjective experiences of reward. We would like to understand how food choice is affected by the rewarding aspects of flavor and taste. The benefits of eating flavorful foods are clear, but we would like to better understand how people incorporate taste into each individual food choice.

\section{Task instructions}

\section{Ratings Task}

In this experiment, you will participate in three tasks. In the first task, you will rate foods on three attributes, one at a time. Ratings will be made using the keyboard. You'll rate foods on their health, how tasty they are to you, and how much you would like to eat them at the end of the experiment. You have the option to rate an item as "neutral", but please avoid that as much as possible. Rate each food based on how it looks on the screen. For example, when rating a picture of a plain piece of bread, tell us how healthful you think it is alone, not how healthful it would have been if it were covered in butter. For each food, you will be shown both an image and a text description. It is important to pay attention to both the image and its text description when rating the foods. This is because later in the experiment you may have to make decisions based on the text descriptions alone.

\section{Baseline Task}

On each trial of this task, you will choose between two different foods. Press the one (1) key to select the food on the left, and the zero (0) key to select the food on the right. Your choices will be displayed as either food images or their text descriptions. The descriptions will be the same text descriptions that you saw in the previous ratings task. At the end of the experiment, you will 
actually receive your food choice from one randomly-selected trial across the entire experiment (from either this task, or next task). You can leave either when you've eaten the food, or when one half hour has expired.

\section{Gambles Task}

In this task, you'll choose between two different baskets. These baskets will be in separate ovals on the right and left sides of the screen, as shown below. Each basket represents a coin flip between the foods in it. In the below example, if you chose the basket on the left, the computer would flip a virtual coin and you would receive the food on top (apple) if it lands on heads, and the food on the bottom (chips) if it lands on tails. To explain this a different way: imagine that you pick one of the two baskets below. You then reach your hand into that basket, and grab one of the two foods at random. Look at the example below.

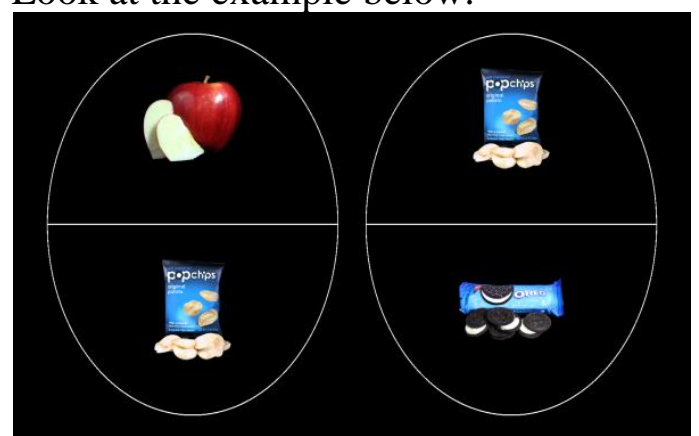

If you chose the basket on the right, the computer would flip a virtual coin. You would receive the food on top (chips) if it lands on heads, and the food on the bottom (Oreos) if it lands on tails. Press the one (1) key to select the basket on the left, and the zero (0) key to select the basket on the right. At the end of the experiment, you will receive a food from one trial in this task, or the previous task. If the selected trial is from this task, the computer will take the basket you picked and flip a virtual coin to determine which of the two foods you will receive. You can leave either when you've eaten the food, or when one half hour has expired. Again, you will be asked to pick a basket with two foods in it. You will have an equal chance of receiving either of the two foods in that basket. Please remember you will only receive ONE of the two foods in your chosen basket, not both foods. 


\section{Simulation Dataset}

To derive simulated decision weights for Figure S5, we arbitrarily assign a value of $(5,1)$ for the taste and health of the unique indulgent item, and $(1,5)$ for the taste and health of the unique disciplined item for simplicity. Next, we begin with a neutral common consequence of $(3,3)$ taste and health, and set it to become more extreme in steps of .01. For the common indulgent consequence, the taste and health go from $(3,3)$ to $(5,1)$. For the common disciplined consequence, the taste and health go from $(3,3)$ to $(1,5)$. In this way, we can test how a common consequence could influence value perception as it becomes symmetrically more extreme in either indulgence or discipline. See Figure S5A for a plot displaying the simulation taste-health value pairs used.

To derive predictions of disciplined and indulgent choices from $\mathrm{CI}$ to $\mathrm{CD}$ conditions, we created 100 simulated unique food pairs whose taste and health ratings ranged from 1 to 5 pulled from a uniform random distribution using MATLAB's rand. In each trial, the unique disciplined food was higher in health and lower in taste rating than the unique indulgent food. Each unique food's value was then normalized (Eq. 2) separately for a CI food that was low in health (1 or 2) and high in taste (4 or 5) and for a CD food that was high in health (4 or 5) and low in taste (1 or 2) according to equation 2. We also applied a decision weight for taste and health (see "Estimating weights and noise parameters" below for this procedure). This was done so that the normalized values account both for an individual's underlying preference for taste and health and for the noise they exhibited in their choices. (Note that the direction of the effects and their statistical significance hold when assuming values of 1 for the decision weights and for noise.)

Next, the proportion of disciplined choices a participant would make was estimated using the normalized and weighted values and a softmax function that included the participant's noise parameter estimated from the Baseline task (Eq. 4). This was done separately for 1) 100 trials with CI foods, 2) 100 trials with CD foods, and 3) with no CC (i.e., using ratings alone and no third food).

Estimating weights and noise parameters. Three free parameters were estimated for each participant: the weight placed on taste, the weight placed on health, and noise ( $\sigma$ in Eq. 4). Baseline task ratings and choices were used to estimate these parameters. This was done by performing a grid search of all possible combinations of possible weights within this range, informed by previous work in the lab: $\sigma=(0.4,4)$ in .2 increments; taste weight $=(-0.5,3)$ in .1 increments; health weight, $(-0.8,2)$ in .1 increments. For each possible combination, the predicted choices and actual choices were compared and the combination that best fit the observed data was used, as assessed by minimizing the negative log likelihood. 


\section{Ratings and Normalization Model Selection}

To assess the predictive performance of the normalization model in the Gambles Task, two choice models were compared. The models differed only in the method used to value the unique food options on each trial. The first model uses only weighted participant ratings to influence choice. The second model uses weighted normalized values to guide choice.

To factor in a participant's preference for health and taste in their choices, we first estimated this using a mixed-effects regression. This estimated the weight of taste and health on normalized taste and health ratings in the Baseline task, which provided per-subject decision weights for taste and health $\left(\mathrm{w}_{\mathrm{i}}\right)$. Next, these weights were used to scale the taste and health values on each trial in the $\mathrm{CC}$ task according to the following equations (in which $\mathrm{S}$ is the set of $\mathrm{y}$ and $\mathrm{z}$ ), and $\mathrm{n}$ is the total number of attributes (here, 2):

Model 1:

Model 2:

$$
v(x)=\sum_{i=1}^{n} w_{i} x_{i}
$$

$$
v(x)=\sum_{y \in S} \sum_{i=1}^{n} \frac{w_{i} x_{i}}{x_{i}+y_{i}}
$$

Next we used maximum likelihood estimation to assess which of two value functions are better predictive of choice: one, the weighted sum of taste and health of each unique food, or two, the weighted taste and health values summed using the normalization function above. For both models, the probability of choosing the gamble with unique food $\mathrm{x}$ was calculated using the softmax function:

$$
p(x \mid x, y, z)=\frac{e^{v(x ; y, z)}}{e^{v(x ; y, z)}+e^{v(y ; x, z)}}
$$




\section{Supplemental Results}

\section{Baseline Disciplined Choices Changed by Prime Condition}

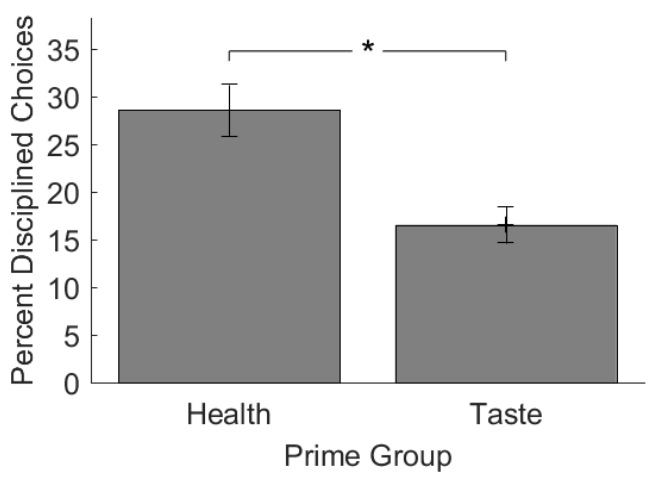

Fig. S1. Percentages of healthy choices in Baseline image-image Conflict Trials, those trials in which one food was tastier but less healthy than the other (separated by prime group). The observed significant effect $(\mathrm{t}(69)=1.19, \mathrm{p}=0.02)$ indicates that the priming of health goals increased the likelihood of a disciplined choice. Error bars represent standard error of the mean computed across participants. 


\section{Common Consequence Disciplined Choices Relative to Baseline}

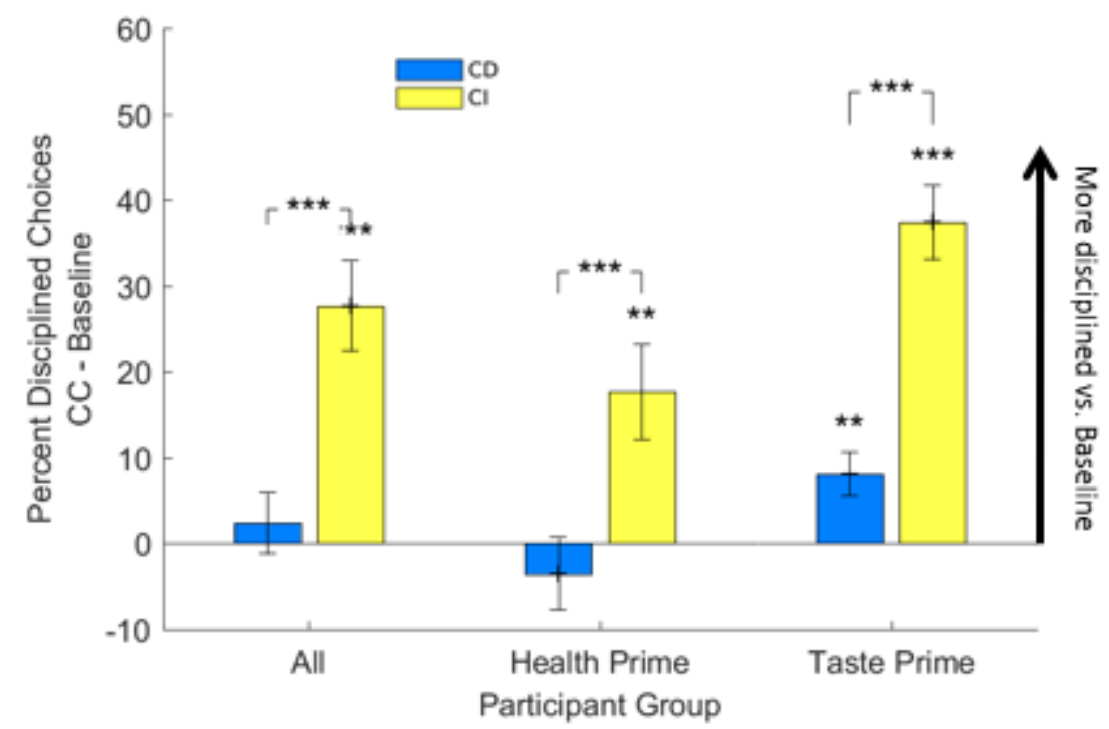

Fig. S2. In this plot, data from Figure 3 are normalized with respect to the proportion disciplined choices in the Baseline task. The CI condition led to significant increases in disciplined choices for both priming groups, whereas the $\mathrm{CD}$ condition only had a significant (and much smaller) effect in individuals who had been primed to emphasize taste goals. Error bars represent standard error of the mean computed across participants. Significance indicators of $* *$ and $* * *$ represent $\mathrm{p}<.01$ and .001 respectively. 


\section{Individual Shifts in Disciplined Choices between Common Consequence Conditions}
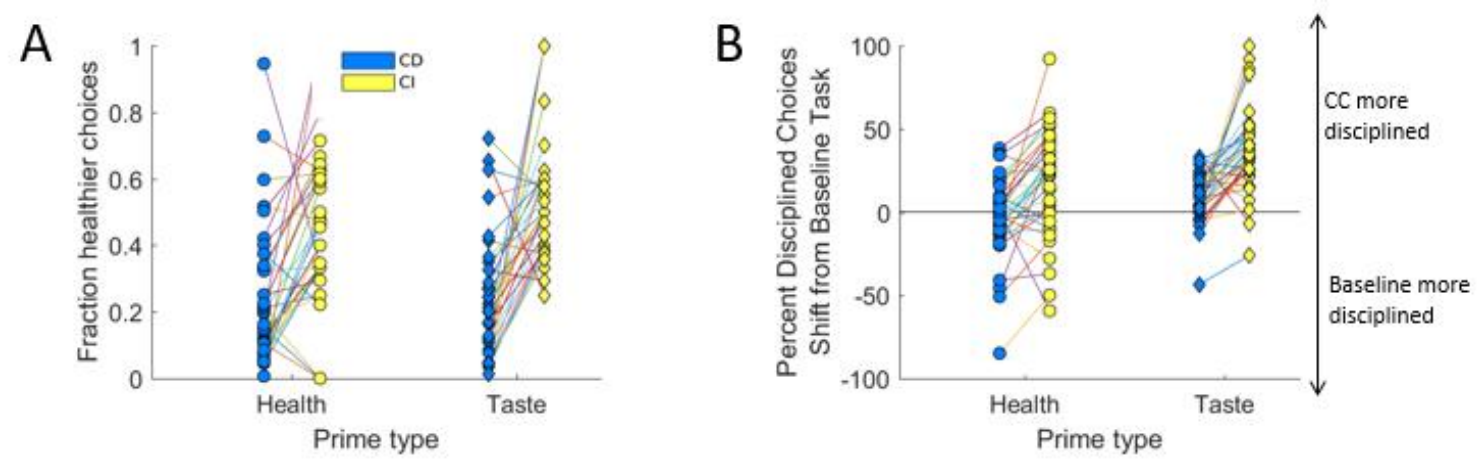

Fig. S3. Percent healthy choices in Common Disciplined and Indulgent conditions, by individual. In plot (a), each data point represents the percentage of healthy choices in the Common Disciplined (CD; blue) and Common Indulgent (CI; yellow) conditions. Lines connect each individual's behavior across CD and $\mathrm{CI}$ conditions. In plot (b), each data point represents the change in percentage of healthier choices from the Baseline task. 


\section{Percent Disciplined Choices by Condition Including NCC trials}

On most CC trials, the Gambles Task featured one food that was in both gambles - that is, it was a common consequence. Some trials used two foods matched on their health and taste ratings (and wanting ratings, if possible). Because very few trials featured either a non-identical "common" disciplined or indulgent food, these trials were not analyzed independently. However, when adding them to the rest of the common consequence trials, our behavioral effect remains unchanged (see Fig. S4).

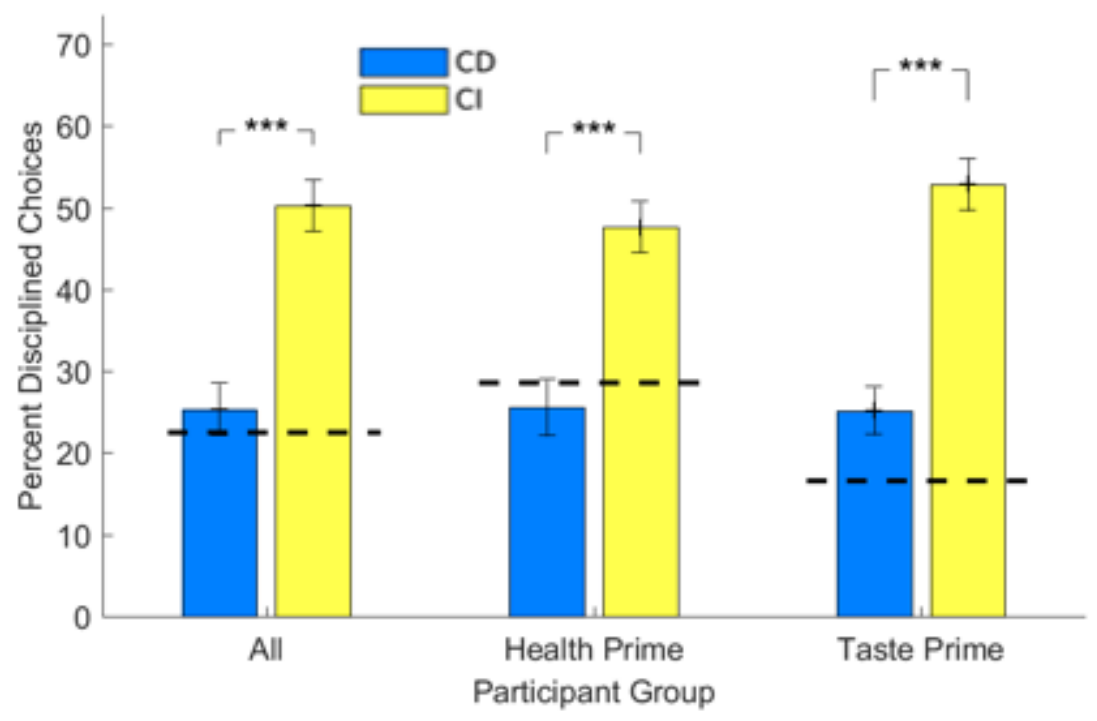

Fig. S4. Effect of common disciplined (CD) and common indulgent (CI) consequences on dietary choices, pooling between identically-matched common consequences, and "common consequences" only approximately matched on their food ratings. The dashed lines show the proportion of disciplined choices in the Baseline task for each group of participants. Error bars represent standard error of the mean computed across participants. Significance indicators of *,**, and *** represent $\mathrm{p}<.05, .01$, and .001 respectively. 


\section{Value Normalization Simulations}

For a range of increasingly-indulgent taste-health pairs (Fig. S1B, from blue to pink), the subjective taste and health of the unique disciplined food improves relative to unique indulgent food, up to a point (Fig. S1B). In contrast, as the common disciplined option becomes more extreme (Fig S1B, from yellow to red), the unique disciplined option decreases in both taste and health. This alone could facilitate healthier choices by reducing the contrast in taste and health such that the less tasty, healthier option becomes more similar to its indulgent counterpart, and allow for preference reversals as the common consequence changes from indulgent to disciplined.

In Baseline trials, that choice can be represented as a point in a two-dimensional space where the axes represent relative value associated with taste and with health (solid circle in Figures S1AB). Introducing a disciplined common consequence (CI) will alter the relative subjective value of those two attributes, moving that choice point along a curve (cool colors). As the CI item becomes increasingly indulgent compared to the unique items, it will increase the subjective health advantage of the disciplined food and decrease the subjective taste advantage of the indulgent food - leading to a greater likelihood of a disciplined choice. Conversely, CD items will reduce the health advantage of disciplined foods and increase the taste advantage of indulgent foods, and thus potentiate indulgent choices. (Note that this simulation also predicts that these effects will disappear when the CC item is extremely different from both unique items, because those items will now seem very similar to each other; see bottom corner of graph in figure S5b.)
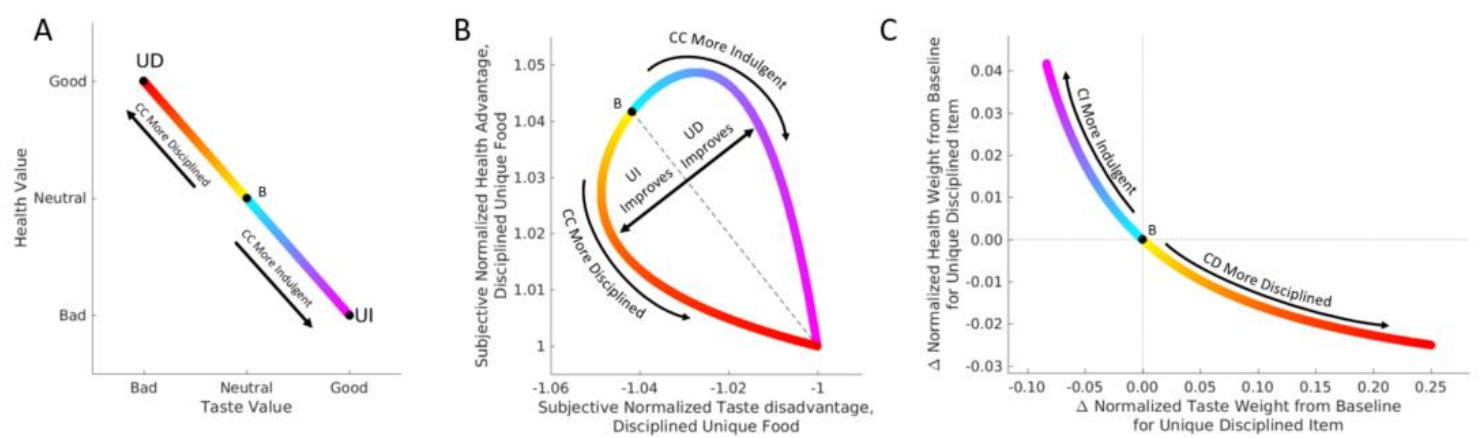

Figure S5. Value normalization with a third option. Plot (a) displays the taste, on the $\mathrm{x}$ axis, and health, on the $\mathrm{y}$ axis, values of the third food (the "Common Consequence", CC) used in the simulation choices. The Common Consequence begins at a neutral value on both taste and health (the black dot labeled B). Then the CC becomes progressively indulgent (tastier and less healthy; cool colormap) or progressively disciplined (healthier and less tasty; warm colormap). The two unique options in the simulated choices (which represent the Gamble Condition's Unique Disciplined (UD) and Unique Indulgent (UI) foods) are also labeled on the graph. In plot (b), the normalized taste of the disciplined unique food relative to the indulgent unique food is plotted as a function of its relative health values. As the common indulgent (CI) consequence becomes increasingly extreme (tastier and less healthy; cool color map), the relative taste difference between the 
indulgent and disciplined food (x-axis) decreases, while its healthful advantage only increases. In contrast, as the common disciplined (CD) consequence gets more extreme (healthier and less tasty; warm color map), the disciplined common consequence decreases in both its taste and health advantage. In both plots, the point at which the cool and warm colors meet (black dot labeled B) is the case in which the Common Consequence is equal in both taste and health (that is, neither health nor taste has an advantage), to represent a case like the Baseline task in which there is no third food. Plot (c) illustrates the change in simulated attribute weights for the unique disciplined item associated with the introduction of a common consequence. As a Common Indulgent (CI) food becomes increasingly tastier and less healthy, the weight placed on the taste of the unique disciplined item decreases, and the weight for health increases (cool colors). Conversely, as a Common Disciplined (CD) becomes increasingly healthy and less tasty (warm colors), the weight placed on the taste of the unique disciplined item increases and the decision weight for health decreases. For comparison, the black dot represents neutrality in both taste and health (cf. the Baseline task, B), and decision weights are normalized relative to this case for ease of reference.

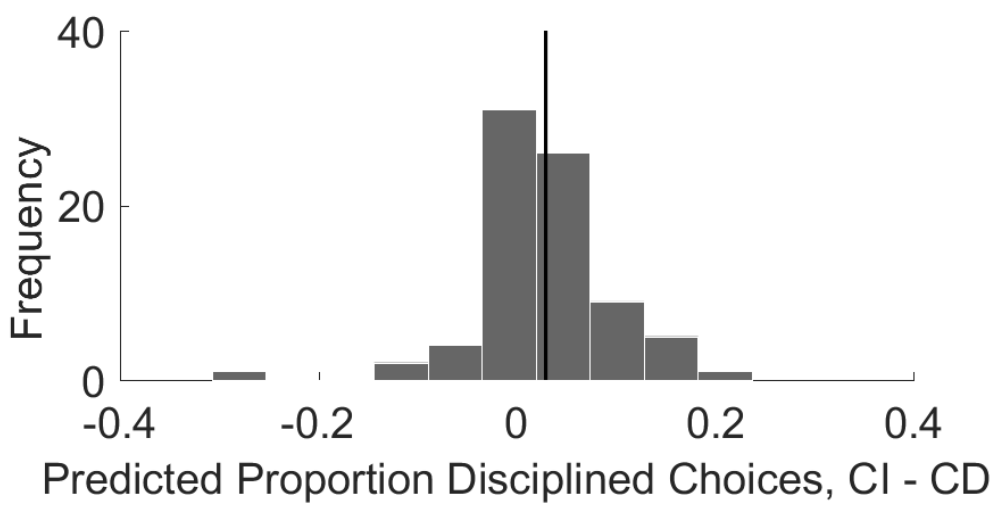

Fig. S6. The distribution of predicted shift in disciplined choices from CI to CD conditions, as predicted by value normalization. The back line plots the mean. 


\section{Common Consequence Decision Weights}

As expected by individuals' overwhelming preference for taste (Sorensen, Moller, Flint, Martens, \& Raben, 2003), taste had a larger weight in choice than did health for both prime groups in both the Baseline Task and in both CC conditions (Figure 5; Baseline task: all participants, $\mathrm{d}=2.40, \mathrm{t}(70)=10.27, \mathrm{p}<1 \times 10^{-10}$; Health prime, $\mathrm{d}=1.71, \mathrm{t}(34)=5.12, \mathrm{p}=1 \times 10^{-}$ 5; Taste Prime, $\mathrm{d}=3.48, \mathrm{t}(35)=10.58, \mathrm{p}<1 \times 10^{-10}$; CI condition: all participants, $\mathrm{d}=2.79, \mathrm{t}(70)$ $=13.05, \mathrm{p}<1 \times 10^{-10}$; Health prime, $\mathrm{d}=2.44, \mathrm{t}(34)=7.74, \mathrm{p}=5 \times 10^{-9}$; Taste Prime, $\mathrm{d}=3.22$, $\mathrm{t}(35)=11.10, \mathrm{p}<1 \times 10^{-10} ; \mathrm{CD}$ condition: all participants, $\mathrm{d}=2.78, \mathrm{t}(70)=12.86, \mathrm{p}<1 \times 10^{-10}$; Health prime, $\mathrm{d}=2.51, \mathrm{t}(34)=7.84, \mathrm{p}=4 \times 10^{-9}$; Taste prime, $\mathrm{d}=3.09, \mathrm{t}(35)=10.59, \mathrm{p}<1 \times 10^{-10}$.

\section{Common Consequence Decision Weights Relative to Baseline}

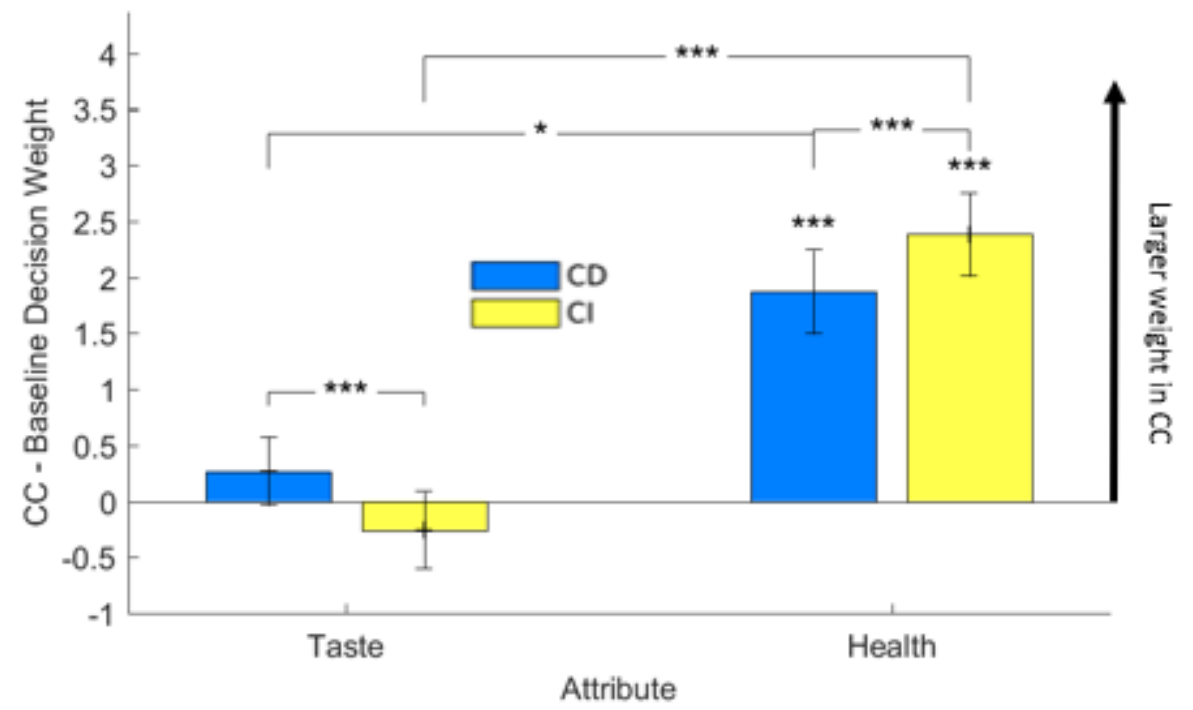

Fig. S7. The shift in taste and health decision weights from the Baseline to CD and CI conditions is plotted in (b), collapsed across participant groups. Error bars represent standard error of the mean computed across participants. Significance indicators of $*, * *$, and $* * *$ represent $\mathrm{p}<.05, .01$, and .001 respectively. 


\section{Influence of Task Order on Behavior}

In this study, the Baseline Task always preceded the CC Task. This was done to minimize concerns about behavioral contamination (e.g., that the Baseline Task could be influenced by the CC Task, preventing us from cleanly estimating the effects of the common consequence). To investigate any potential influence of this task order, we performed the following analysis. We ran a full mixed-effects regression with left option choice predicted by trial food wanting (left minus right, normalized to -1 to 1 ; see later responses for the reason for norming ratings), an indicator for baseline (0) or Common Consequence Task (1), trial number, and wanting*task, wanting*trial, and task $*$ trial interactions.

The results from this analysis show that our observed effects of task are independent of any effects of trial. We found a significant effect of wanting ( slope $=5.46, p=6 \times 10^{-56}$ ), a significant effect of task (slope $=0.44 \mathrm{p}=2 \times 10^{-5}$ ), and no significant effect of trial number $\left(\right.$ slope $=6 \times 10^{-5}$, $\mathrm{p}=.85)$. We also found that wanting was a stronger predictor of choice in the Common Consequence Task (task ${ }^{*}$ wanting slope $=3.74, p=3 \times 10^{-20}$ ). Wanting became less significantly predictive of choice as the trial number increased (trial ${ }^{*}$ wanting slope $=-0.005 \mathrm{p}=2 \times 10^{-9}$ ). The interaction between task type and trial number was not significant (slope $=-0.0001, p=.75$ ). This indicates that wanting had less influence upon choices as trials progressed within a task, but that there was an overall increase in effect of wanting from the first task to the second.

These analyses show that our observed difference between tasks remained significant, even when controlling for trial number, and that that order effects, while important for many phenomena, are not driving the greater influence of wanting on choice in the CC task. 


\section{Influence of Differences in the Difficulty of Making a Disciplined Choice Across Conditions}

To properly evaluate behavioral differences in disciplined choices between conditions, it is important to ensure that the difficulty of making a disciplined choice did not significantly differ between conditions. To ensure this, we performed the following two analyses.

First, we examined whether differences in choices between baseline and CC trials depended on taste, health, and wanting rating differences, used as proxies for choice difficulty. To do this, we first examined whether difference in disciplined choices from the Baseline to CI task (for each individual) was predicted by the mean absolute value of the difference in wanting, taste, and health between options (e.g., mean of $\mid$ Wanting left $_{-}-$Wanting $_{\text {right }} \mid$ for all trials in a given task). We found that there was no significant relationship for any variable (wanting, $\mathrm{p}=.55$; taste, $\mathrm{p}=.51$; health, $\mathrm{p}=.39$ ). Second, we performed the same analysis to ensure that the shift in disciplined choices from CD to CI was not related to the difference in ratings. We again found no significant relationship here (wanting $\mathrm{p}=.42$; taste $\mathrm{p}=.29$; health $\mathrm{p}=.66$ ). Note that because there was no behavioral difference in proportion healthy choices from Baseline to CD trials, we did not conduct this analysis for the different between those trial types.

Second, we attempted to ensure that there were no differences between the critical CI and CD trials on the difficulty of making a healthy choice by examining whether the CI and CD trial conditions were matched for mean difference in rating measures. That is, was the mean value of |Wanting left $_{\text {- Wanting }}$ right (and the other ratings) similar across these two conditions? If so, then condition differences in those rating measures (and associated choice difficulty) could not be drivers of our observed difference in choices. We found no significant differences between these conditions for differences in mean wanting rating $(\mathrm{CI}$ Wanting $=1.66, \mathrm{SD}=0.47$; $\mathrm{CD}$ Wanting $=$ $1.67, \mathrm{SD}=0.46)$, mean taste rating $(\mathrm{CI}$ Taste mean $=1.69, \mathrm{SD}=0.58 ; \mathrm{CD}$ Taste mean $=1.72$, $\mathrm{SD}=0.55)$, or mean health rating $(\mathrm{CI}$ Health mean $=2.21, \mathrm{SD}=0.48 ; \mathrm{CD}$ Health mean $=2.21$, $\mathrm{SD}=0.47$ ). This means that the large-magnitude difference in proportion of healthy choices (cf. Figure 3) cannot be attributed to the ratings of the foods used in each condition.

Further, to ensure that the effect of disciplined choices between CI and CD conditions was not driven by differences in the difficulty of making a disciplined choice between the two conditions, we performed the following analysis. First, ratings for taste and health were normalized from 1 to 5 across all three conditions: Baseline, CD, and CI. Next, taste and health decision weights for these three conditions were estimated as described in the Results section entitled "Attribute decision weights and common consequence: Experimental evidence", using the normalized ratings instead. As shown below (Figure S7), decision weights for the CI and CD conditions are in the same direction as seen without normalization (main text Figure 5) and the differences remain significantly different.

Together, these results indicate that the differences in the weight placed on taste and health between the $\mathrm{CI}$ and $\mathrm{CD}$ conditions are not driven by any differences in the spread of ratings between these conditions. 

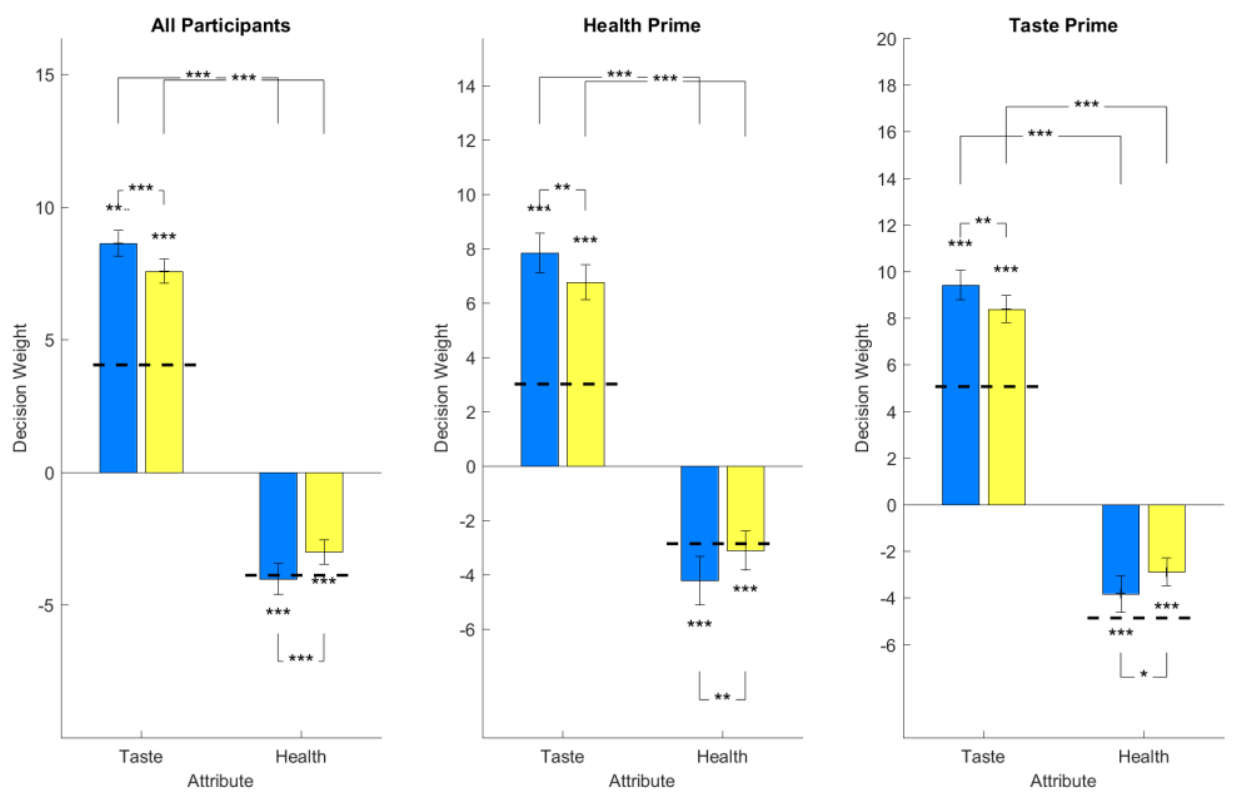

Figure S8. Decision weights by Task and CC condition, estimated using food attribute ratings normalized across all conditions. The bar graph plots the influence of taste and health information, as estimated by logistic decision weights, upon decisions involving common disciplined (CD) or indulgent (CI) common consequences. The dashed lines represent decision weights in the Baseline task for each priming group. For all panels, error bars represent standard error of the mean computed across participants. Significance indicators of $*, * *$, and $* * *$ represent $\mathrm{p}<.05, .01$, and .001 respectively. 